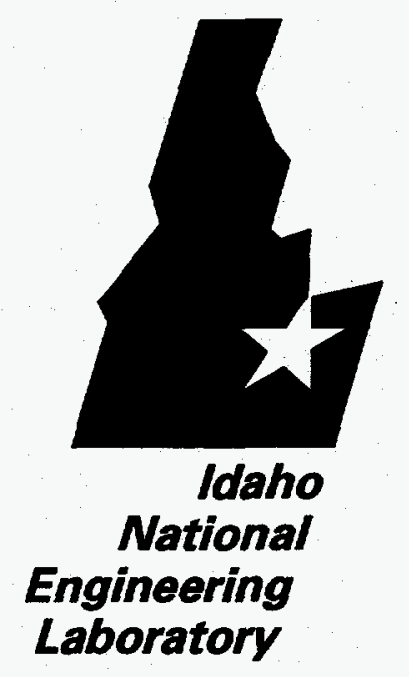

INEL-95/0206

April 1996

Statistical Analysis of
Random Duration Times

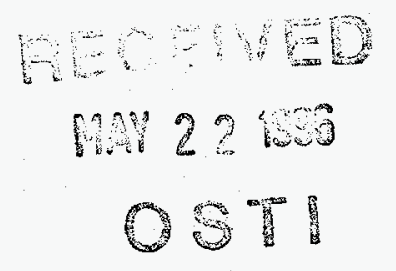

M. E. Enge/hardt

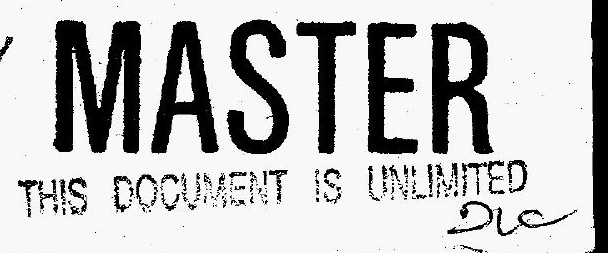




\section{NOTICE}

This report was prepared as an account of work sponsored by an agency of the United States Government. Neither the United Sates Government nor any agency thereof, nor any of their employees, makes any warranty, expressed or implied, or assumes any legal liability or responsibility for any third party's use, or the results of such use, of any information, apparatus, product or process disclosed in this report, or represents that its use by such third party would not infringe privately owned rights. 


$$
\text { INEL- } 95 / 0206
$$

INEL-95/0206

\title{
Statistical Analysis of Random Duration Times
}

\author{
M. E. Engelhardt
}

Published April 1996

\section{Idaho National Engineering Laboratory \\ Idaho Falls, Idaho $\mathbf{8 3 4 1 5}$}

Prepared for the

U.S. Nuclear Regulatory Commission

Office for Analysis and Evaluation of Operational Data

Reliability and Risk Analysis Branch

Under DOE Idaho Operations Office

Contract DE-AC07-94ID13223 


\section{DISCLAMMER}

Portions of this document may be illegible in electronic image products. Images are produced from the best available original document. 


\begin{abstract}
This report presents basic statistical methods for analyzing data obtained by observing random time durations. It gives nonparametric estimates of the cumulative distribution function, reliability function and cumulative hazard function. These results can be applied with either complete or censored data. Several models which are commonly used with time data are discussed, and methods for model checking and goodness-of-fit tests are discussed. Maximum likelihood estimates and confidence limits are given for the various models considered. Some results for situations where repeated durations such as repairable systems are also discussed.
\end{abstract}

FIN E8247-Special Methods and Databases: Statistical Methods 


\section{SUMMARY}

This report deals primarily with statistical models and methods for analyzing data obtained by observing random time durations. Common examples are failure times of components and systems, as well as repair times for repairable systems.

Nonparametric estimates and confidence procedures are given for the cumulative distribution function, the reliability function and the cumulative hazard function. Some basic model checking procedures such as hazard plotting and goodness-of-fit tests are presented and illustrated by application to operational data.

Some parametric models which are commonly used with time data such as the exponential, Weibull, normal and lognormal models are presented. Goodness-of-fit tests which are adapted specifically for use with these models are discussed and illustrated by application to data. Maximum likelihood estimates and confidence limits for the parameters, the cumulative distribution function and the reliability function are also considered. 


\section{FOREWORD}

The Office for Analysis and Evaluation of Operational Data (AEOD) of the U.S. Nuclear Regulatory Commission (NRC) is continually reviewing data from U.S. commercial nuclear power plants. Statistical analysis forms an important part of this work. Accordingly, the Reliability and Risk Analysis Branch of AEOD has asked the Idaho National Engineering Laboratory (INEL) to write a series of reports presenting the appropriate statistical tools for the kinds of data most commonly encountered. These reports are being written in parallel with a series of studies on the performance of safety systems in nuclear power plants, and they reflect the influence of those studies.

The reports are expected to provide tools and guidance to analysts of NRC data, although the statistical methods can, by their nature, be applied to data from many other fields. The reports are intended to quickly help a new user. The report on collecting operational data should be understandable by anyone with a technical background, although the examples are slanted towards an engineer with nuclear experience. The reports on statistical methods should be readable and immediately usable by a person with training in statistics but with no experience analyzing such data. Some of the reports, of necessity, are more advanced than others; for example, the report on loglinear modeling will be easier to understand if the reader has first assimilated the reports on binomial data, Poisson data, and linear models. Nevertheless, the reports are all intended to be introductory to the extent possible, suitable as brief self-study texts to help readers move quickly to the tasks of data collection and analysis. In addition, the reports should be usable as texts or references in short courses for persons with less training.

The first reports written or planned in this series are

Collecting Operational Event Data for Statistical Analysis, September 1994, EGG-RAAM-10086, by Corwin L. Atwood

Hits per Trial: Basic Analysis of Binomial Data, September 1994, EGGRAAM-11041, by Corwin L. Atwood

Events in Time: Basic Analysis of Poisson Data, September 1994, EGGRAAM-11088, by M. E. Engelhardt 
Modeling Patterns in Continuous Data Using Linear and Related Models DRAFT, 1995, INEL-95/0120, by M. E. Engelhardt (Final version planned for 1996)

Modeling Patterns in Count Data Using Loglinear and Related Models, December 1995, INEL-95/0121, by Corwin L. Atwood

Statistical Analysis of Random Duration Times, April 1996, INEL95/0206, by M. E. Engelhardt

Practical Guidance for Statistical Analysis of Operational Event Data, October 1995, INEL-95/0234, by Corwin L. Atwood 


\section{ACKNOWLEDGMENTS}

Cindy Gentillon supplied some of the data used in the examples. Cory Atwood and Harry Martz provided insightful review comments which helped to improve the overall presentation. 


\section{CONTENTS}

ABSTRACT $\ldots \ldots \ldots \ldots \ldots \ldots \ldots \ldots \ldots \ldots \ldots \ldots \ldots \ldots \ldots \ldots$

SUMMARY $\ldots \ldots \ldots \ldots \ldots \ldots \ldots \ldots \ldots \ldots \ldots \ldots \ldots \ldots$

FOREWORD $\ldots \ldots \ldots \ldots \ldots \ldots \ldots \ldots \ldots \ldots \ldots \ldots \ldots \ldots \ldots$

ACKNOWLEDGMENTS $\ldots \ldots \ldots \ldots \ldots \ldots \ldots \ldots \ldots \ldots \ldots$

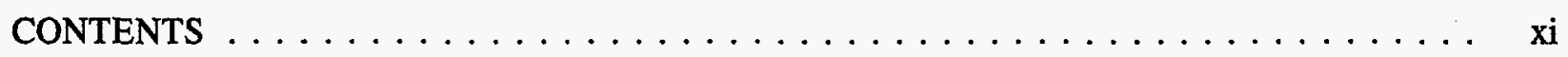

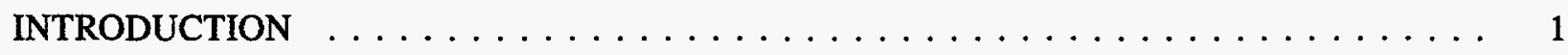

MODELS FOR DURATION TIMES $\ldots \ldots \ldots \ldots \ldots \ldots \ldots \ldots \ldots \ldots$

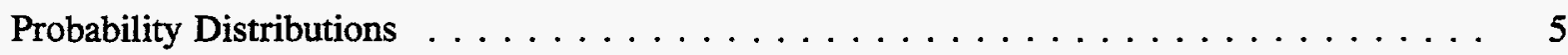

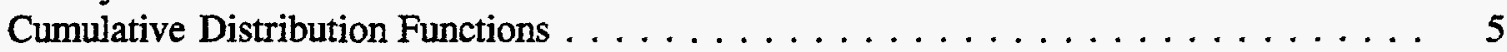

Probability Density Functions . . . . . . . . . . . . . . . . . . . . 6

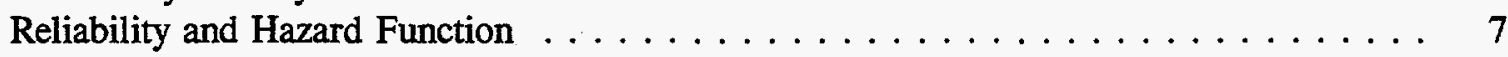

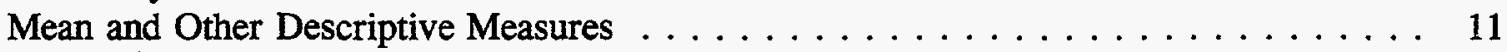

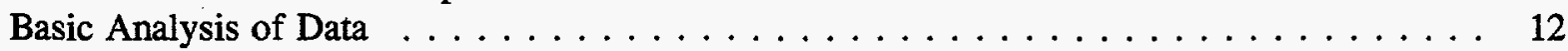

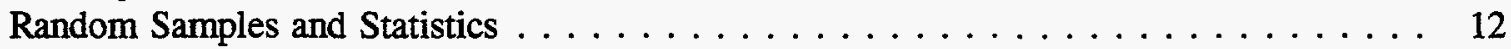

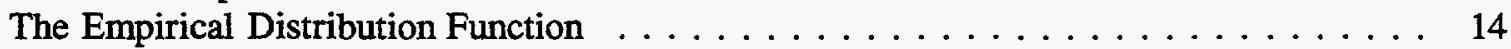

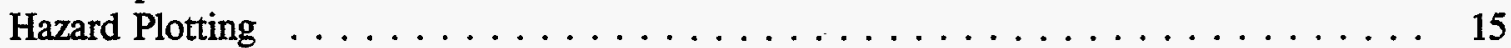

Goodness-of-Fit Testing $\ldots \ldots \ldots \ldots \ldots \ldots \ldots \ldots \ldots \ldots \ldots$

Some Special Distributions . . . . . . . . . . . . . . . . . . . . . . . . 19

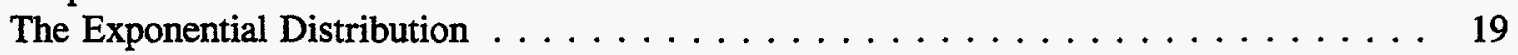

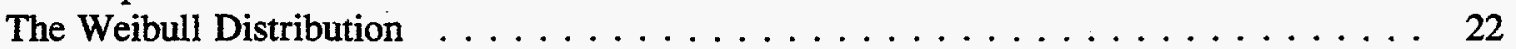

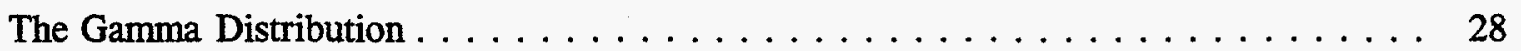

The Normal Distribution . . . . . . . . . . . . . . . . . . . 30

The Lognormal Distribution . . . . . . . . . . . . . . . . . . 34

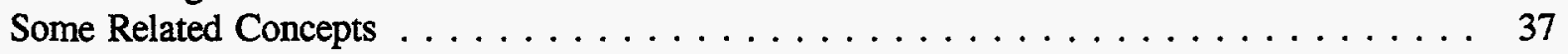

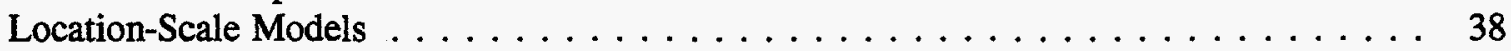

Probability Plotting . . . . . . . . . . . . . . . . . . . . 39

Transformations . . . . . . . . . . . . . . . . . . . . . 39

Estimation Using SAS $\ldots \ldots \ldots \ldots \ldots \ldots \ldots \ldots \ldots \ldots \ldots$

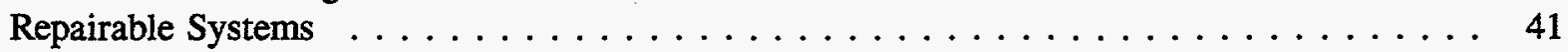

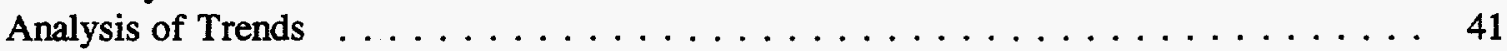

Availability and Maintainability $\ldots \ldots \ldots \ldots \ldots \ldots \ldots \ldots$

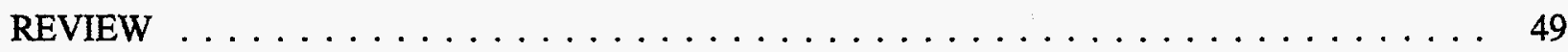

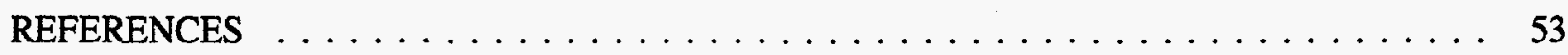

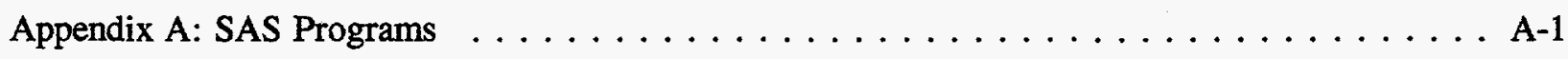

Appendix B: Mathematical Details $\ldots \ldots \ldots \ldots \ldots \ldots \ldots \ldots \ldots \ldots$ 
Estimation of Reliability from Censored Data $\ldots \ldots \ldots \ldots \ldots \ldots \ldots$

REFERENCES $\ldots \ldots \ldots \ldots \ldots \ldots \ldots \ldots \ldots \ldots \ldots \ldots \ldots \ldots \ldots \ldots \ldots$

\section{FIGURES}

Figure 1. Probability density function and cumulative distribution function. . . . . . . . . 6

Figure 2. The reliability function, hazard function and cumulative hazard function. . . . . . 10

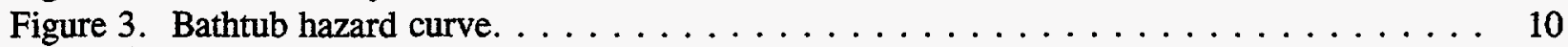

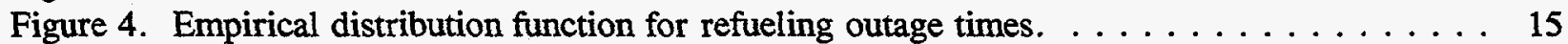

Figure 5. Comparison of EDF based estimate and Nelson's estimate of the cumulative hazard

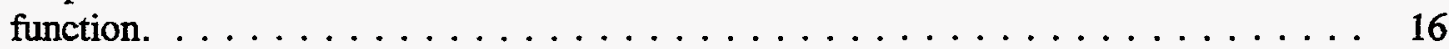

Figure 6. Probability density, cumulative distribution and hazard functions for the exponential

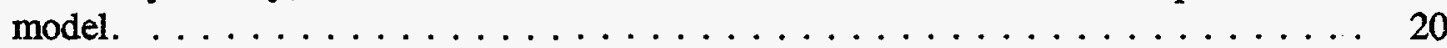

Figure 7. Cumulative hazard plot for outage-time data using linear scales. . . . . . . . . 21

Figure 8. Weibull probability density functions for different shape parameter values $\beta$. . . . . 22

Figure 9. Weibull hazard functions for different shape parameter values $\beta \ldots \ldots \ldots \ldots 23$

Figure 10. Cumulative hazard plot of refueling times with $\log -\log$ scale. $\ldots \ldots \ldots \ldots \ldots$

Figure 11. Gamma probability density functions for different shape parameter values $\kappa . \ldots \ldots 28$

Figure 12. Gamma hazard functions for different shape parameter values $\kappa$. . . . . . . . . . 29

Figure 13. Standard normal probability density, cumulative distribution and hazard functions. . . 32

Figure 14. Lognormal probability density functions for different combinations of $\gamma$ and $\delta$. . . 34

Figure 15. Hazard function of the lognormal distribution for different combinations of $\gamma$ and $\delta$. . 35

Figure 16. Number of critical compressor failures as a function of times (operating days). . . . 42

Figure 17. Comparison of availability for repaired system and nonrepaired system. . . . . . . 47

Figure 18. Uptime and downtime status for Plant A during the period 1985-94 . . . . . . . . . 47

\section{EXAMPLES}

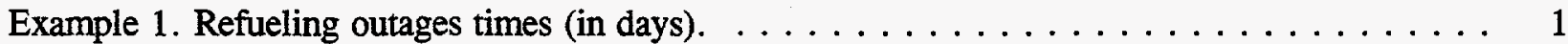

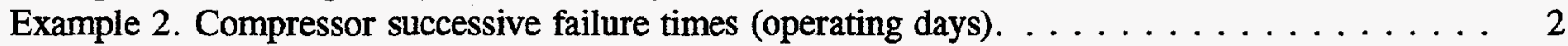

Example 3. Operating cycles with alternate uptimes and downtimes. . . . . . . . . . 3 


\section{Statistical Analysis of Random Duration Times}

\section{INTRODUCTION}

This report is about modeling and analysis of time data and applications with operational data. Often in an experiment or study, a measurement of interest is a random time or duration. Most of the key results involving random times were motivated by the study of lifetimes, and therefore much of this report will focus on those results. For lifetimes of people or organisms, such analysis is usually called survival analysis, and for failure times of hardware or systems, it is called analysis of reliability. These topics are often discussed in different parts of the literature, but they have much in common. Although we emphasize the reliability aspects in this report, the discussion here will also apply to more general studies of duration.

We will first consider three examples which are based on operational data.

\section{EXAMPLES}

Example 1. Refueling outages times (in days).

Nuclear power plants must be shut down periodically for refueling. The times between a plant shutting down for refueling and being restarted and put back into operation tend to vary. This refueling time will be affected by random factors such as the time it takes for refueling and the time required for other procedures such as periodic surveillance tests which are usually done during refueling shutdowns. The following times are the refueling outage times for two plants located at the same station for the years 1985-1994.

Plant A

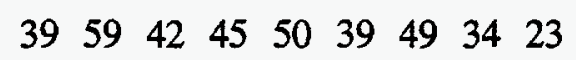
Plant B
$\underline{ }$
$\begin{array}{llllllll}52 & 63 & 47 & 46 & 63 & 39 & 48 & 32\end{array}$

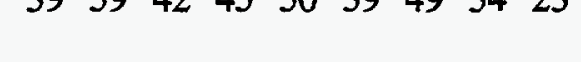

For a second example, we consider 90 successive failure-times (operating days) for a specific compressor at a power plant, as reported by Høyland and Rausand (1994). These data were collected during the period 1968 until 1989. 
Example 2. Compressor successive failure times (operating days).

The data in this example are successive failure times (operating days) for a specific compressor. All failures of this compressor were classified as either critical or noncritical failures. A critical failure, in this context, was defined as a failure which required shutting down the compressor to make the repair. Noncritical failures were ones that could be corrected without the compressor needing to be shut down. For example, noncritical failures included situations such as instrument failures, and failures of the seal oil system and the lubrication oil system which could be repaired while the compressor continued to run. The data presented here represent the critical failures.

\begin{tabular}{rrrrrrrrrrr}
\hline 1.0 & 4.0 & 4.5 & 92.0 & 252.0 & 277.0 & 277.5 & 284.5 & 374.0 & 440.0 \\
444.0 & 475.0 & 536.0 & 568.0 & 744.0 & 884.0 & 904.0 & 1017.5 & 1288.0 & 1337.0 \\
1338.0 & 1351.0 & 1393.0 & 1412.0 & 1413.0 & 1414.0 & 1546.0 & 1546.5 & 1575.0 & 1576.0 \\
1666.0 & 1752.0 & 1884.0 & 1884.2 & 1884.4 & 1884.6 & 1884.8 & 1887.0 & 1894.0 & 1907.0 \\
1939.0 & 1998.0 & 2178.0 & 2179.0 & 2188.5 & 2195.5 & 2826.0 & 2847.0 & 2914.0 & 3156.0 \\
3156.5 & 3159.0 & 3211.0 & 3268.0 & 3276.0 & 3277.0 & 3321.0 & 3566.5 & 3573.0 & 3594.0 \\
3640.0 & 3663.0 & 3740.0 & 3806.0 & 3806.5 & 3809.0 & 3886.0 & 3886.5 & 3892.0 & 3962.0 \\
4004.0 & 4187.0 & 4191.0 & 4719.0 & 4843.0 & 4942.0 & 4946.0 & 5084.0 & 5084.5 & 5355.0 \\
5503.0 & 5545.0 & 5545.2 & 5545.5 & 5671.0 & 5939.0 & 6077.0 & 6206.0 & 6206.5 & 6305.0
\end{tabular}

In Example 2, the times represent cumulative operating times of the same compressor. Repair times between failures and subsequent restarts are not considered in this example. Consequently, the data represent successive failure times of the compressor, all measured from the initial startup time of the compressor.

For repairable systems, times between failures are often modeled as independent and identically distributed random variables, corresponding to an assumption that a failed unit is repaired to "like new" condition, or replaced with a new unit. Such a model is usually called a renewal process. However, there are situations in which the renewal assumption is not correct, and times between failures exhibit a trend. For example, because critical failures result in a temporary loss of compressor function, repair actions might involve corrective measures intended to reduce the likelihood of this failure mode occurring 
If such repairs are successful, a trend toward longer times between failure would be expected over a period of time. This situation is referred to as reliability growth. On the other hand, a system which receives minimal repairs when it fails might show a trend toward shorter times between failure.

For a third example, we consider a situation in which there are two types of durations, times during which a system is operating (up) and times when the system is not operating (down).

Example 3. Operating cycles with alternate uptimes and downtimes.

An operating plant is up and running at some time $t=0$, such as the low power license date. The plant then operates for a period of time until it has to shut down for refueling or some other cause. After a period of being down, the plant then becomes operational again, and the cycle starts over again. This cycle is repeated over and over again during the life of the plant. Thus, there would be two sequences of durations; uptimes and downtimes, denoted by $t_{\mathrm{i}}$ and $d_{\mathrm{i}}$, respectively. If these durations are random, it is impossible to know in advance whether the plant is up or down at some future time $t$. It would be desirable to know the probability that the plant will be up (available) at time $t$ or down (unavailable) at time $t$. The following table gives the uptimes and downtimes (rounded to the nearest day) for specific plant between the dates 10/01/85 and $01 / 31 / 94$.

\begin{tabular}{lllllllllllll}
\hline$t_{\mathrm{i}}:$ & 194 & 320 & 314 & 318 & 320 & 63 & 256 & 195 & 134 & 3 & 299 & 275 \\
$d_{\mathrm{i}:}:$ & 37 & 57 & 40 & 43 & 48 & 7 & 37 & 2 & 47 & 4 & 32 & 21
\end{tabular}

The data sources used in these examples are partly proprietary and not necessarily of the highest quality. Therefore, plants are not identified by name. The data in the first and third examples come from internal NRC studies. 


\section{MODELS FOR DURATION TIMES}

Much of the literature which is relevant to duration times focuses on analyzing lifetimes and repair times of components and systems. Consequently, most of our discussion will be about such applications, although these results can be applied to general duration times. For convenience we will refer to either a system or component as a "unit" for which a time measurement is obtained. Such a measurement will be modeled as a random quantity. An example of this is the time to failure or lifetime of a unit. In other words, the elapsed time from when the unit starts operation $(t=0)$ until it fails. Typically, the time to failure is subject to chance variations. This might be true for many diverse reasons, such as design flaws or manufacturing defects in the hardware, human errors in the installation or operation of equipment, or environmental reasons. It is therefore natural to interpret the time to failure as a random variable $T$. Similarly, other duration times such as repair times are subject to chance variations and should be modeled as random.

\section{Probability Distributions}

There are different ways to specify a probability distribution, and in this section we will discuss some of the various concepts; how to interpret them, and how they are related to each other. The usual convention is to denote the random variables as capitals, $T$, and observed values as lower case, $t$.

\section{Cumulative Distribution Functions}

The Cumulative Distribution Function (CDF) of a real-valued random variable $T$ is defined as

$F(t)=P[T \leq t]$

for all real numbers $t$. The name is sometimes abbreviated to distribution function. The CDF is the probability that the random variable $T$ will assume a value which is either less than or equal to $t$. It is well known (see e. g. Bain and Engelhardt, 1992, p. 60) that the a CDF has certain mathematical properties. For example, the CDF is a monotonic increasing function of $t$, and also $F(t) \rightarrow 0$ as $t \rightarrow-\infty$ and $F(t) \rightarrow 1$ as $t \rightarrow+\infty$. An abbreviated notation for these limiting properties is to write $F(-\infty)=$ 0 and $F(+\infty)=1$.

The CDF provides a common way of characterizing a probability distribution, but there are also other characterizations available. Because our focus is on durations, it can be assumed that observed values will be positive valued. Consequently, it can be assumed that $F(t)=0$ for all $t \leq 0$ and that the 
property $F(-\infty)=0$ can be replaced with the property $F(0)=0$. If there is a positive probability that a unit has already failed upon installation, then $F(0)=P[T \leq 0]>0$. This would result in a discontinuity at $t=0$. In the following discussion we will assume that the CDF is continuous for all $-\infty<t<+\infty$, including $t=0$.

\section{Probability Density Functions}

A function $f(t)$ is a probability density function (pdf) for a continuously distributed positive-valued random variable $T$ if

$$
F(t)=\int_{0}^{t} f(u) d u
$$

The variable $u$ is a dummy variable of integration, and $t$ is the upper limit of the integral. An example of a pdf and the associated CDF are shown in Figure 1.

The pdf is commonly used to characterize the distribution of lifetimes (or durations) of a whole population of units. The population might be a large set of identical components which are operating in similar applications and with durations which vary due to random causes. Even if there is only one such unit in existence, it is possible to think of a "conceptual" population of identical units but with random durations.

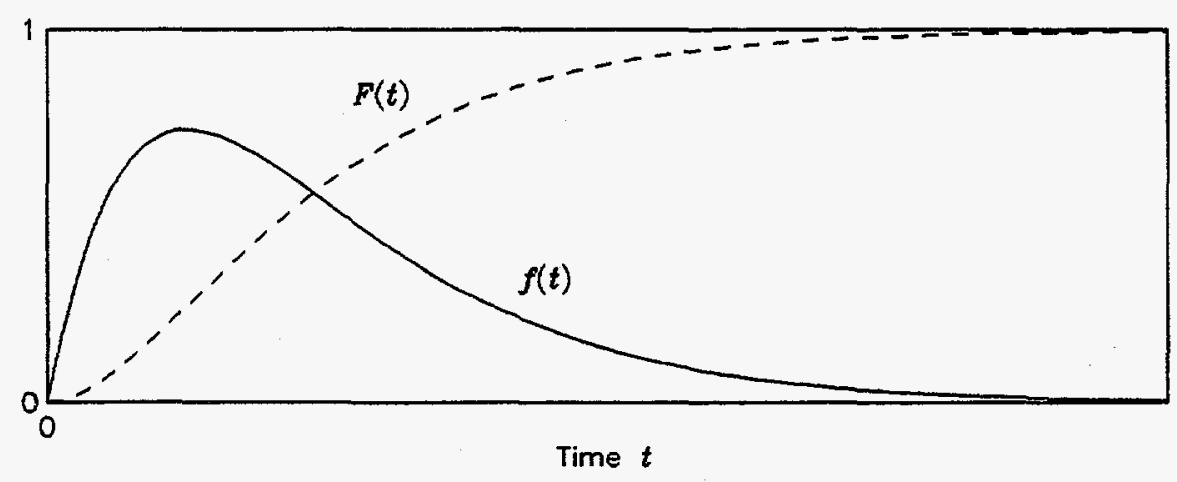

Figure 1. Probability density function and cumulative distribution function.

It is clear that pdf can be obtained from the CDF by differentiation 


$$
f(t)=\frac{d}{d t} F(t)
$$

for all $t>0$ at which the derivative exists. It follows that probabilities corresponding to occurrences in a small interval of time are approximately proportional to the pdf,

$P[t<T \leq t+\Delta t] \approx f(t) \Delta t$.

Therefore, the ordinate of a pdf has units of "probability density" and not probability (as for a CDF). Thus, a pdf determines how to assign probability over small intervals of time. Now consider an arbitrary interval $(a, b]$. In the limit approximating the interval $(a, b]$ with a union of arbitrarily small intervals, it follows that

$$
P[a<T \leq b]=\int_{a}^{b} f(t) d t
$$

There are also characterizations which have special interpretations for time-to-failure distributions. In particular, we will next consider the reliability and hazard functions.

\section{Reliability and Hazard Function}

The reliability function of a unit is defined as

$$
R(t)=P[T>t]
$$

Hence, $R(t)$, called the reliability at time $t$, is the probability that the unit does not fail in the time interval $(0, t]$ or equivalently, the probability that the unit is still operating at time $t$. When used as a reliability criterion, it is common to state a time, say $t_{0}$, called the mission time, and require for a unit that the reliability at mission time $t_{0}$ be at least some prescribed level, say $R_{0}$. For example, a pump might be required to operate successfully for at least 12 hours with probability at least 0.99 . The requirement, in this case is then $R_{0}=0.99$ and $t_{0}=12$. In terms of the reliability function, this would mean $R(12) \geq$ 0.99 . One interpretation would be that such a pump would perform for the required mission time for 99 percent of the situations when it is called on to do so. Another interpretation is that 99 percent of all such pumps would perform as required. In survival analysis, the reliability function is called the survivor function, and the notation used in this setting is $S(t)$.

Another common way to characterize reliability is in terms of the hazard function. Suppose when we know that the unit is still operating at time $t$, we are concerned with the probability it will fail in a 
small interval of time $(t, t+\Delta t]$. This is the conditional probability $P[t<T \leq t+\Delta t|T\rangle t]$, which can also be written as

$$
P[t<T \leq t+\Delta t \mid T>t]=\frac{P[t<T \leq t+\Delta t]}{P[T>t]}=\frac{F(t+\Delta t)-F(t)}{R(t)} .
$$

If we divide this conditional probability by the length of the interval $\Delta t$ and take the limit as $\Delta t \rightarrow 0$, we get a function $h(t)$, called the hazard function. In particular, we have

$$
h(t)=\lim _{\Delta t \rightarrow 0} \frac{P[t<T \leq t+\Delta t \mid T>t]}{\Delta t}=\lim _{\Delta t \rightarrow 0} \frac{F(t+\Delta t)-F(t)}{\Delta t} \frac{1}{R(t)}=\frac{f(t)}{R(t)}
$$

which implies that when $\Delta t$ is small,

$P[t<T \leq t+\Delta t \mid T>t] \approx h(t) \Delta t$.

Expression (2) is analogous to (1), except that the probability is conditional on the unit having survived until $t$, whereas (1) is relative to all units in the original population, either still surviving or not. Suppose a large number, say $N$, of identical units are put into operation at time $t=0$, and $n$ is the number which fail in the interval $(t, t+\Delta t]$. It follows that $f(t) \Delta t \approx n / N$, the observed relative frequency of units failed in the interval $(t, \Delta t]$. On the other hand, if we denote by $N_{t}$ the number of the original $N$ units which are still in operation at time $t$, then $h(t) \Delta t \approx n / N_{t}$, the observed relative frequency of surviving units which fail in this same interval. Thus, $f(t)$ is a measure of the risk of failing at time $t$ for any unit in the original set, whereas $h(t)$ is a measure of the risk of failing at time $t$, but only for units which have survived this long.

The hazard function is used as a measure of "aging" for units in the population. If $h(t)$ is an increasing function, then units are aging or wearing out with time. Of course, in general the hazard function can exhibit many types of behavior other than increasing with time, and we will discuss other possible behaviors later in the report. In actuarial science the hazard function is called the force of mortality, and it is used as a measure of aging for individuals in a population. More generally, the hazard function gives an indication of the "proneness to failure" of a unit after time $t$ has elapsed. Other terms which are also used instead of hazard function are hazard rate and failure rate. However, the term failure rate is often used in other ways in the literature of reliability (see Ascher and Feingold, 1984, p. 19).

There are some interesting and useful connections between the hazard function and the reliability 
function. We note, in particular that

$$
f(t)=\frac{d}{d t} F(t)=\frac{d}{d t}[1-R(t)]=-R^{\prime}(t)
$$

and therefore

$$
h(t)=-\frac{R^{\prime}(t)}{R(t)}=-\frac{d}{d t} \ln R(t)
$$

Because $R(0)=1$, we have

$$
H(t) \equiv \int_{0}^{\tau} h(u) d u=-\ln R(t)
$$

The function $H(t)$ is called the cumulative hazard function, and it follows that

$$
R(t)=\exp \left(-\int_{0}^{t} h(u) d u\right)=\exp (-H(t))
$$

The reliability function, $R(t)$, and the CDF, $F(t)=1-R(t)$, are therefore uniquely determined by the hazard function, $h(t)$, and the pdf can be expressed as

$$
f(t)=h(t) \exp \left(-\int_{0}^{t} h(u) d u\right)=h(t) \exp (-H(t))
$$

Figure 2 shows the reliability, hazard and the cumulative hazard function for the example of Figure 2.

The hazard function in Figure 2 is an increasing function of time which would be consistent with units with a dominant wearout effect for the entire life of the unit. It is often the case that manufactured units will exhibit different aging behavior over three different periods of their lifetime. Typically the hazard starts out high in the initial period. This can be explained by the presence of undiscovered manufacturing defects which cause early failures called infant failures. Such defective units are difficult to find, and can only be discovered by pre-testing the units during a "burn-in" phase. When a unit has survived the infant mortality phase, the hazard function often stabilizes and remains relatively constant for a certain amount of time until it starts to increase as the unit begins to wear out. This situation is illustrated in Figure 3. The graph of such a hazard function is usually called a bathtub curve or U-shaped curve.

From the shape of the bathtub curve, the lifetime of a unit may be divided into three typical intervals: the burn-in period, the random or chance failure period and the wear-out period. During the 


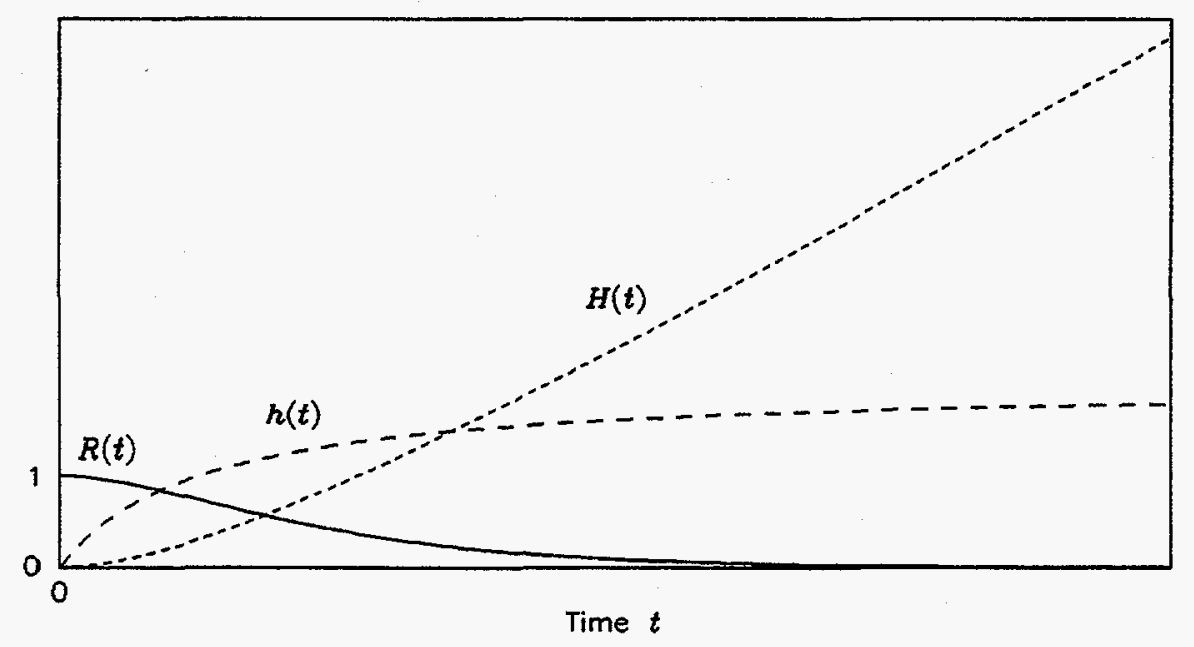

Figure 2. The reliability function, hazard function and cumulative hazard function.

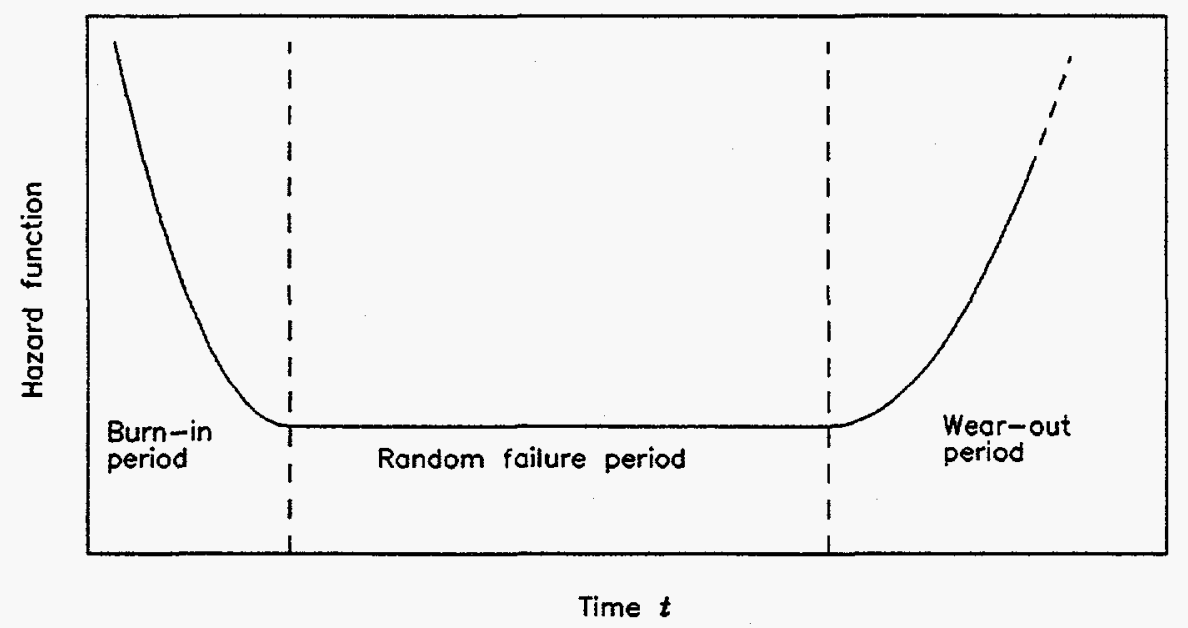

Figure 3. Bathtub hazard curve.

useful period, the dominant cause of failures is "random" failures. For example, units might fail due to external causes such as power surges or other environmental factors rather than problems attributable to 
the defects or wear-out in the units. This example is somewhat idealized because for many types of units the hazard function will tend to increase slowly during the later stages of the chance failure period. This is particularly true of mechanical components and systems which consist largely of mechanical components. On the other hand, for many electrical components such as transistors and other solid-state devices, the hazard function remains fairly flat once infant failure period is over.

\section{Mean and Other Descriptive Measures}

It is desirable to have a set of numerical values which describe important characteristics of the distribution. If we denote a random duration time by $T$, the mean or expected value of $T$ is defined as

$$
E(T)=\int_{0}^{\infty} t f(t) d t
$$

Other common notations for the mean are $\mu$ and $\mu_{T}$. For example, reliability criteria are often expressed in terms of the mean time to failure (MTTF). More generally, we will call this the mean duration or simply the mean.

In the reliability setting, if it is the policy to replace a unit or repair it to "like-new" condition immediately upon failure, and the replacement or repair time is negligible, with respect to the MTTF, then the MTTF is also called the mean time between failures (MTBF). If the replacement or repair time is not negligible, then the MTBF also must take this into account. This situation will be discussed in greater detail later in a section about recurrent events such as successive failures of a repairable system.

There is a connection between $E(T)$ and the reliability function. As noted previously, the pdf and reliability are related as $f(t)=-R^{\prime}(t)$. When $E(T)<\infty$, we can use this property together with integration by parts in (3), to obtain

$$
E(T)=\int_{0}^{\infty} R(t) d t
$$

It is sometimes easier to determine $E(T)$ by this formula than by (3). This also provides a mathematical reason why a unit with a uniformly higher reliability will have a longer MTTF.

It is also important to have a numerical measure of the amount of variability from one observed failure time to the next. The variance of $T$ is defined as

$$
\operatorname{var}(T)=E\left[(T-\mu)^{2}\right]=\int_{0}^{\infty}(t-\mu)^{2} f(t) d t
$$


Another useful form is

$\operatorname{var}(T)=E\left(T^{2}\right)-\mu^{2}$

and another common notation for the variance is $\sigma^{2}$. The standard deviation $\sigma$ (or $\sigma_{T}$ ) is a related measure of variability which has the advantage of being expressed in the same units as $T$.

In some applications, it is convenient to use distribution quantiles. The pth quantile (or $100 p$ th percentile) of a distribution with $\operatorname{CDF} F(t)$ is the solution $t_{p}$ of the equation

$p=F\left(t_{p}\right)$

The median is the 0.5 th quantile, or the value $t_{\text {med }} \equiv t_{0.05}$ such that half of the population values lie above and the other half lie below it. For a distribution which is symmetric about its mean, the mean and median are the same. However, duration time distributions are typically nonsymmetric and skewed to the right with the median below the mean.

Another descriptive measure which is often of interest is the mode which is defined as a value $t_{\text {mode }}$ which maximizes the pdf. In other words, $f\left(t_{\text {mode }}\right) \geq f(t)$ for all $t$.

\section{Basic Analysis of Data}

It is necessary to first consider some mathematical details relating to how the data are obtained. The most common assumption is that the data is obtained by random sampling.

\section{Random Samples and Statistics}

A random sample of size $n$ from a population with pdf $f(t)$ is defined as a set of random variables $T_{1}, T_{2}, \ldots, T_{n}$ such that

- The $T_{i} \mathrm{~s}$ are independent,

- Each $T_{i}$ has the pdf $f(t)$.

The term random sample also refers to the observed values or data, $t_{1}, t_{2}, \ldots, t_{n}$. Typically population 
distributions have unknown parameters such as a population mean $\mu$ or a population variance $\sigma^{2}$.

A statistic or estimator of an unknown population parameter is a known function of a set of observable random variables. In the present setting, the set of observable random variables is a random sample. Two important examples of statistics are the sample mean, defined as

$$
\bar{T}=\frac{1}{n} \sum_{i=1}^{n} T_{i}
$$

and the sample variance, defined as

$$
S^{2}=\frac{1}{n-1} \sum_{i=1}^{n}\left(T_{i}-\bar{T}\right)^{2}
$$

The sample mean and sample variance are known to be unbiased for the population mean and variance, respectively. In other words, $E(\bar{T})=\mu$ and $E\left(S^{2}\right)=\sigma^{2}$. The same functions applied to data are called estimates, denoted by $\bar{t}$ and $s^{2}$. For example, if we combine the refueling time data from the two plants in Example 1, we have a sample mean of $\bar{t}=45.3$ days with a sample variance of $s^{2}=114.85$ and a sample standard deviation of $s=10.7$ days. Thus, an estimate of the mean number of days for a refueling outage is about 45 days, and an estimate of the standard deviation is about 11 days.

The sample mean and sample variance can be used to estimate the population mean and variance, but there are cases in which population parameters other than the mean and variance are of interest. In such cases, it is desirable to have a general approach for finding estimates. The method of maximum likelihood (ML) provides a rather general way of deriving estimates in many situations. For a random sample from a population with pdf $f(t ; \theta)$, with parameter $\theta$, the likelihood function is the product $L(\theta)$ $=\Pi_{j} f\left(t_{i} ; \theta\right)$. The ML estimate is the value $\hat{\theta}$ which maximizes $L(\theta)$ for a given set of data. If the distribution depends on more than one parameter, then the ML approach can be generalized by taking $\theta$ to be a vector of the unknown parameters. The method of ML can also be applied in situations where the data is not from a random sample, using as the likelihood function the joint pdf $L(\theta)=f\left(t_{1}, t_{2}, \ldots\right.$, $\left.t_{n} ; \theta\right)$. More discussion of the method of ML can be found in many books, including Bain and Engelhardt (1992).

Some analyses require that the data first be ranked or put in ascending order from the smallest to the largest observation. The variables obtained by ordering the random sample are called the order statistics denoted by $T_{(1)}<T_{(2)}<\cdots<T_{(n)}$, and the ordered data is denoted in a similar manner, but with lower case notation $t_{(1)}<t_{(2)}<\cdots<t_{(n)}$. Some important estimates based on the order statistics 
are the sample median, and the sample range. The sample median is defined as the "middle" order statistic $t_{(m)}$ if $n$ is odd with $m=(n+1) / 2$, and the average of the two middle order statistics $\left(t_{(m)}+\right.$ $\left.\mathrm{t}_{(m+1)}\right) / 2$ if $n$ is even with $m=n / 2$. A more general definition which is sometimes used with an even sample size is to let the sample median be any value in the interval $\left[t_{(m)}, t_{(m+1)}\right]$ which would include the average of the endpoints as one possible solution. The more general definition has the disadvantage that the sample median is not unique. The sample range is the difference between the largest and smallest ordered observations, $t_{(n)}-t_{(1)}$. The sample median and sample range are sometimes used as alternatives to the sample mean and sample standard deviation as measures of central tendency and dispersion in the sample. The sample median is an estimate of the population median $t_{\text {med }}$, and under some circumstances an estimate of the population standard deviation can be based on the sample range. In particular, for some special distributions it is possible to find a constant $d_{n}$ such that $\left(T_{(n)}-T_{(1)}\right) / d_{n}$ is an unbiased statistic for $\sigma$. For the combined data of Example 1, the sample median and sample range are 46 days and $(63-23)=40$ days, respectively.

There are situations in which some of the times are not observed. For example, suppose $n$ light bulbs are put on test and the failure times are recorded as they occur. The resulting failure times would be ordered, and an appropriate way to represent the data would be as observed values of order statistics. It is fairly common to have a situation in which the last few failure times are not observed due to a time constraint or possibly due to some limitation in the measuring process. If some of the data are not observed, the sample is said to be censored. For example, if it is decided in advance of taking observations that only those which occur before some fixed time $t_{0}$ will be recorded and used in the analysis, they the data are said to be time censored (usually called type I censoring). With this kind of censoring, the number of failures prior to the censoring time $t_{0}$ will be random and part of the data set. If a fixed number $r<n$ of failure times are recorded before ceasing observation, then the data are said to be failure censored (usually called type II censoring). Another type of censoring known as interval censoring occurs when no exact times are known but instead only a count of the number of failures in an interval is known. Most of the procedures discussed here are for complete (uncensored) samples, but more discussion of censoring and estimation with censored data are given in Appendix B.

\section{The Empirical Distribution Function}

An estimate of $F(t)$ called the empirical distribution function (EDF) is defined as follows: For an arbitrary value of $t>0$, we define

$\hat{F}(t)=$ (Number of observations $\leq t) / n$.

A related estimate called the empirical reliability function $(\mathrm{ERF})$ is $\hat{R}(t)=1-\hat{F}(t)$. The EDF and ERF 
are step functions. The EDF increases by $1 / n$ at each observed time if all observations are distinct, and the ERF decreases in a similar manner. More generally, if there are $m$ times equal to $t, \hat{F}(t)$ has a positive jump of $m / n$ at $t$, and the ERF has a similar drop. It is possible to show that the EDF and ERF are unbiased estimators, respectively, of the CDF and reliability functions. Also, each of these functions closely approximates its population counterpart when $n$ is large.

For a numerical example we consider the refueling outage times in Example 1, the $n=17$ ordered times are: $23,32,34,39,39,39,42,45,46,47,48,49,50,52,59,63$ and 63 . The time 39 occurs three times, and 63 occurs twice. With continuous variables such as time, a repeated value should not happen, but these times are rounded to the nearest day, which means that repeated values should not be surprising. Thus, the EDF for these data will have jumps of size $3 / 17$ at $t=39$ and $2 / 17$ at $t=63$, and all other jumps are of size 1/17. The graph of this EDF is given in Figure 4.

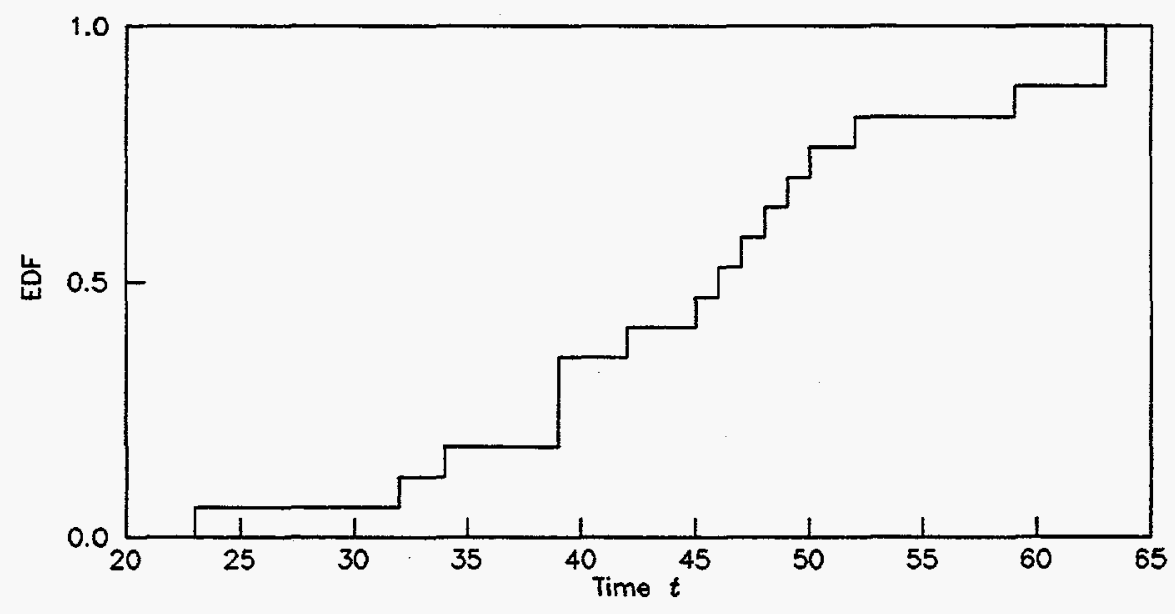

Figure 4. Empirical distribution function for refueling outage times.

\section{Hazard Plotting}

It is possible to estimate the cumulative hazard function using these results because of the relationship between $R(t)$ and $H(t)$, which suggests the following EDF or ERF based estimate:

$$
\hat{H}(t)=-\log [\hat{R}(t)]=-\log [1-\hat{F}(t)]
$$

The graph of this estimate of the cumulative hazard function is a step function which takes jumps at the 
data values. The estimate is 0 for $t$ less than the smallest observed time, and it is undefined for $t$ greater than or equal to the largest observed time because $\hat{F}(t)=1$ in this range.

There is another way, due to Nelson (1972), of estimating the cumulative hazard function from the duration times. It is possible to compute directly from the duration times an "empirical" estimate of $H(t)$. For example, suppose $n$ units are tested until they fail, and a failure occurs at time $t$. If $k$ units have survived until time $t$ (including the one which just failed), then $1 / k$ is the observed proportion failing at $t$ among the $k$ units which have survived until time $t$. Consequently, $1 / k$ is an estimate of $h(t)$, and the sum of all such estimates for failure times $t_{i} \leq t$ provides an estimate of $H(t)$. Unlike the estimate based on the EDF, Nelson's estimate is defined at the largest observed time, and it can also be used to estimate $H(t)$ when the data are censored. This latter approach to estimating $h(t)$ and $H(t)$ gives a first-order approximation to the estimate based on $\hat{R}(t)$, and it is slightly easier to compute. A comparison of the EDF based cumulative hazard estimate and Nelson's estimate is given in Figure 5.

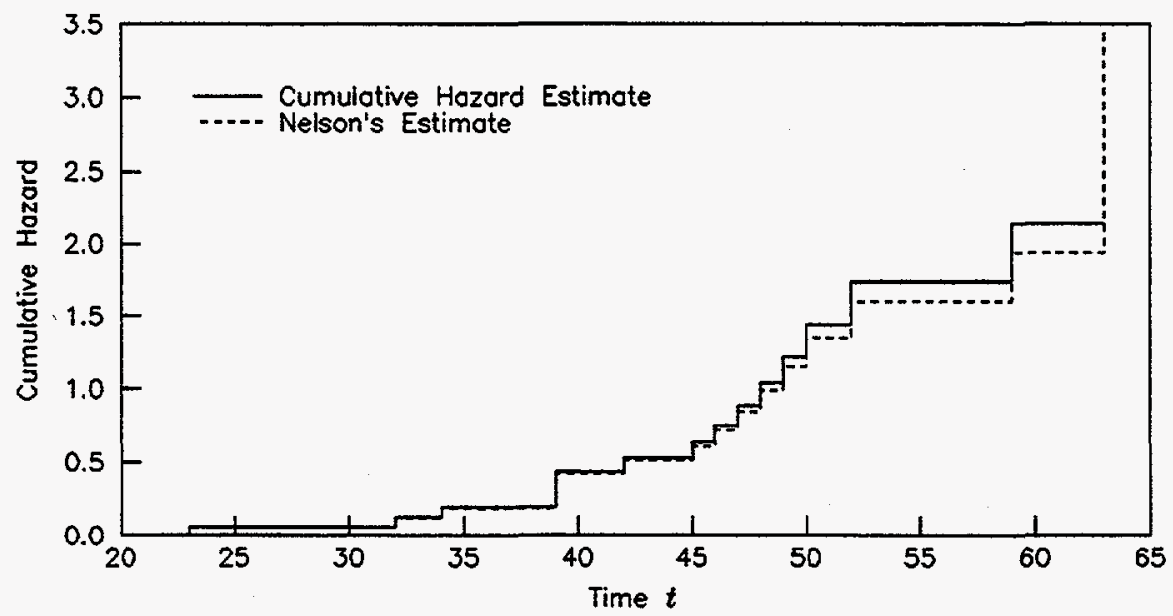

Figure 5. Comparison of EDF based estimate and Nelson's estimate of the cumulative hazard function.

For practical purposes, either of the two approaches can be used, although they are not identical. Either approach gives a nonparametric way to estimate reliability for times within the range of the data. For example, the estimate of $R(35)$ using the EDF is $\hat{R}(35)=1-\hat{F}(35)=1-3 / 17=0.82$. For comparison, Nelson's estimate of $H(35)$ is -0.188 , giving $\exp (-0.188)=0.83$ as an estimate of $R(35)$.

Another approach, which applies with censored data, uses a more general estimator known as the Kaplan-Meier product limit estimator. This is discussed in Appendix B. 
An advantage of the EDF-based estimate is that confidence limits can be easily computed. This is because the variable $Y=n \hat{R}(t)$ has a binomial distribution with parameters $n$ and $p=R(t)$. Confidence limits for the parameter $p$ of a binomial distribution are discussed by Atwood (1994). Using this approach, limits for a conservative $90 \%$ confidence interval for $R(35)$ are $R_{\mathrm{L}}(35)=0.60$ and $R_{\mathrm{U}}(35)=$ 0.93. A SAS program for computing confidence limits for the parameter $p$ of a binomial distribution is given in Appendix A of Atwood (1994).

As mentioned previously, much of the focus with duration times is on reliability. Because it is important to demonstrate high reliability for a product, lower confidence limits for reliability are generally emphasized more than upper confidence limits. In other words, it is important to demonstrate that lifetimes tend to be long. In the case of refueling outage times, the reverse is true because it is important to a utility's financial health that a plant not be down longer than necessary. Thus, from the utility's perspective it is important to demonstrate that refueling outage times tend to be short. Thus, an upper confidence limit on $F(t)$ would be of interest in such an application. However, the results on reliability are useful here because if $R_{\mathrm{L}}(t)$ is a lower confidence limit for $R(t), F_{\mathrm{U}}(t)=1-R_{\mathrm{L}}(t)$ is an upper confidence limit for $F(t)$. Thus, from the above results, we can say that $1-0.60=0.40$ is an upper $95 \%$ confidence limit on $F(35)$, the probability that a refueling outage time will not exceed 35 days. In other words, we are $95 \%$ confident that on the average at most 4 out of every 10 outage times for refueling will exceed 35 days.

It is possible to develop a graphical analysis in which some appropriate transformation of the failure times $t_{i}$ are plotted versus some appropriately transformed estimate of $H\left(t_{i}\right)$, where the particular transformation used depends on the particular model under consideration. For example, if a Weibull distribution is under consideration, then the logarithm of both $t_{i}$ and the estimate of $H\left(t_{i}\right)$ is the appropriate transformation (see the discussion of the Weibull distribution in the next section). With such an analysis, if the plotted points lie approximately on a straight line, the corresponding distribution is a good candidate for fitting to the data. Next we discuss another way which is often used to check whether a particular model is an appropriate choice for a given set of data.

\section{Goodness-of-Fit Testing}

The data from a random sample of observed times are contained in the interval of positive real numbers, $(0, \infty)$. Suppose we divide the interval $(0, \infty)$ into $c$ subintervals, or cells, say $A_{1}, A_{2}, \ldots$, $A_{c}$, and let $p_{j}=P\left[T \in A_{j}\right]$, the probability that the random time $T$ is contained in the $j$ th cell if $T$ is distributed as $F(t)$, a completely specified CDF with no unknown parameters. Then, for a random sample of size $n$, let $o_{j}$ represent the observed number of times in the $j$ th cell, and let $e_{j}=n p_{j}$, the expected number of times in the $j$ th cell. The chi-square goodness-of-fit statistic is 


$$
\chi^{2}=\sum_{j=1}^{c} \frac{\left(o_{j}-e_{j}\right)^{2}}{e_{j}}
$$

An approximate size $\alpha$ test of the null hypothesis $\mathrm{H}_{0}: T \sim F(t)$ would reject $\mathrm{H}_{0}$ if $\chi^{2}>\chi_{1-\alpha, p}^{2}$, which is the $(1-\alpha)$ th quantile of the $\chi^{2}$ distribution with $\nu=c-1$ degrees of freedom. This approximate distribution is appropriate when $n$ is large, and when the expected cell counts are sufficiently large. A commonly used rule for the large expected cell counts is $e_{j} \geq 5$. However, as noted by Engelhardt (1994, p. 15) this rule is very conservative. In particular, for a $5 \%$ level test with equiprobable cells, a more reasonable requirement is $n / c \geq 1$, and for a $1 \%$ level test the requirement is $n / c \geq 2$.

If the distribution being tested involves one or more unknown parameters, then the $\chi^{2}$ test can be modified in the following way: (1) Estimate the parameters using the ML estimates based on the cell counts $o_{1}, o_{2}, \ldots, o_{c}$ and (2) adjust the degrees of freedom, $\nu=c-m-1$, where $m$ is the number of unknown parameters which are estimated.

There are also goodness-of-fit tests which make use of the actual times rather than counts within intervals such as the case with the chi-square goodness-of-fit test. Tests which are known as EDF tests are based on the difference between a hypothesized distribution $F(t)$ and the EDF $\hat{F}(t)$. A well-known EDF test called the Kolmogorov-Smirnov test is based on the maximum absolute difference $|F(t)-\hat{F}(t)|$. However, another EDF test which is easier to compute is the Cramer-Von Mises (CVM) test. If the CDF is $F(t ; \theta)$ where $\theta$ is either an unknown parameter of a vector of unknown parameters, with ML estimate $\hat{\theta}$, then the CVM statistic is

$$
C M=\frac{1}{12 n}+\sum_{i=1}^{n}\left[F\left(t_{(i)} ; \hat{\theta}\right)-\frac{i-0.5}{n}\right]^{2} .
$$

This statistic is based on the fact that when $T \sim \mathrm{F}(\mathrm{t} ; \theta)$ then quantities $\mathrm{F}\left(\mathrm{t}_{(1)} ; \theta\right), \mathrm{F}\left(\mathrm{t}_{(2)} ; \theta\right), \ldots, \mathrm{F}\left(\mathrm{t}_{(n)} ; \theta\right)$ are distributed as uniform order statistics on the interval $(0,1)$, and, differences $\mathrm{F}\left(\mathrm{t}_{(i)} ; \theta\right)-(i-0.5) / n$ tend to be small. Consequently, a large value of $C M$ leads to a rejection of the null hypothesis $\mathrm{H}_{0}: T \sim$ $\mathrm{F}(\mathrm{t} ; \theta)$. Thus, $\mathrm{H}_{0}$ is rejected at the $\alpha$ level of significance if $C M>C M_{1-\alpha}$, the $(1-\alpha)$ th quantile of $C M$ under $\mathrm{H}_{0}$. For cases where tables are available for the CVM, it is preferable to the chi-square test. Tables for computing the quantiles of $C M$ are given by Bain and Engelhardt (1992, p.613) for some special distributions which we shall discuss next. Some additional results on EDF tests are given by D'Agostino and Stephens (1986, Ch. 4). Examples of these tests will be given in the next section. 


\section{Some Special Distributions}

There are several special distributions which are commonly used in modeling lifetimes and more generally random durations. Each of these distributions has properties which make them reasonable models for certain situations. We will now discuss some of these distributions and their properties.

\section{The Exponential Distribution}

A random variable $T$ has the exponential distribution with parameter $\lambda>0$ if its pdf has the form

$f(t)=\lambda \exp (-\lambda t)$

for $t>0$, and zero otherwise. The reliability function is

$R(t)=\exp (-\lambda t)$

The expected value and variance for the exponential distribution are

$E(T)=1 / \lambda$

and

$\operatorname{var}(T)=1 / \lambda^{2}$

Another parameter which is sometimes used in the mean $\theta=1 / \lambda$.

The hazard function is

$h(t)=\lambda \exp (-\lambda t) / \exp (-\lambda t)=\lambda$

It should be noted that the hazard function is constant and has the value $\lambda$. This property is unique to the exponential distribution and it means that a unit which has the exponential life distribution does not wear out. Thus, if a unit is truly exponentially distributed, the bathtub curve would be flat over the entire life of the unit, with no infant phase and no wearout period. As noted previously, solid-state devices typically behave in this manner, although, due to manufacturing defects, an adequate burn-in period might be required. 
Another well known property of the exponential model is the "memoryless" or "no memory" property. In particular, it is not difficult to show that

$P[T>t+a \mid T>a]=P[T>t]$

for all $a>0$ and $t>0$. It can be shown that the exponential model is the only continuous life model which has this property. The interpretation of the no memory property is similar to the constant hazard property. In particular, is says that an old unit which has not failed by age $a$ is as reliable as a brand new unit. As an example, suppose a RAM chip in a computer has been in operation for 100 hours. If the chip has not failed, then in terms of reliability it would make no difference whether the chip is left in service or replaced with a new one. This assumes that the chips have been through an adequate burn-in phase to eliminate defective chips. This property is related to the constant hazard property.

The probability density function, cumulative distribution function and hazard function for an exponential distribution are shown in Figure 6.

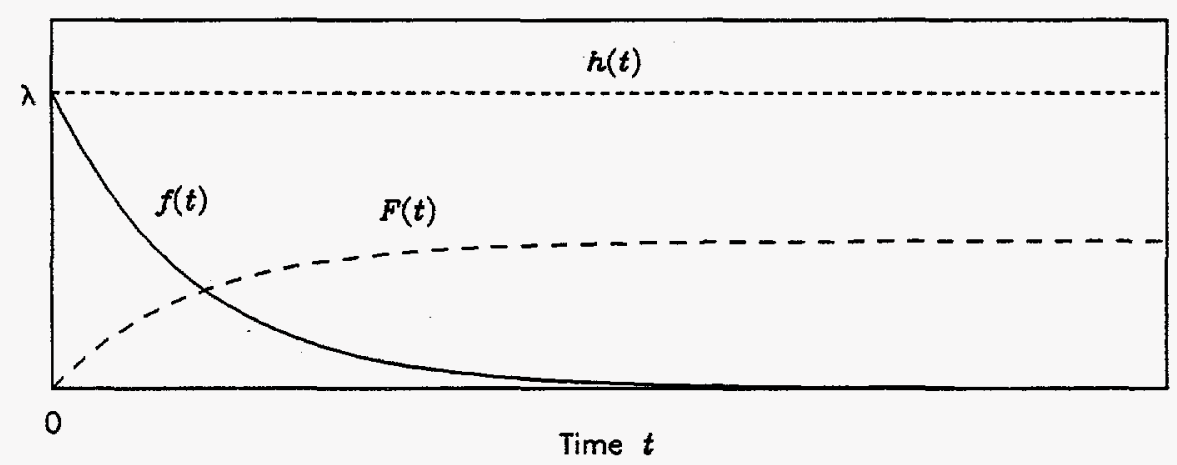

Figure 6. Probability density, cumulative distribution and hazard functions for the exponential model.

The ML estimate for $\lambda$ is the reciprocal of the sample mean, $\hat{\lambda}=1 / \bar{t}$. It is possible to compute upper and lower confidence limits for $\lambda$ based on the ML estimate and chi-square percentiles. Namely, for a $(1-2 \alpha) 100 \%$ confidence interval, the lower and upper limits are respectively

$\lambda_{\mathrm{L}}=\chi_{\alpha, 2 \pi}^{2} /(2 n \bar{t})$

and

$\lambda_{U}=\chi_{1-\alpha, 2 \pi}^{2} /(2 n \bar{t})$. 
The graphical method of hazard plotting is particularly convenient with the exponential distribution because the cumulative hazard function is $H(t)=\lambda t$, so that plotted points will tend to cluster about a straight line with intercept 0 . Such a plot is shown in Figure 7 for the data of Example 1. The

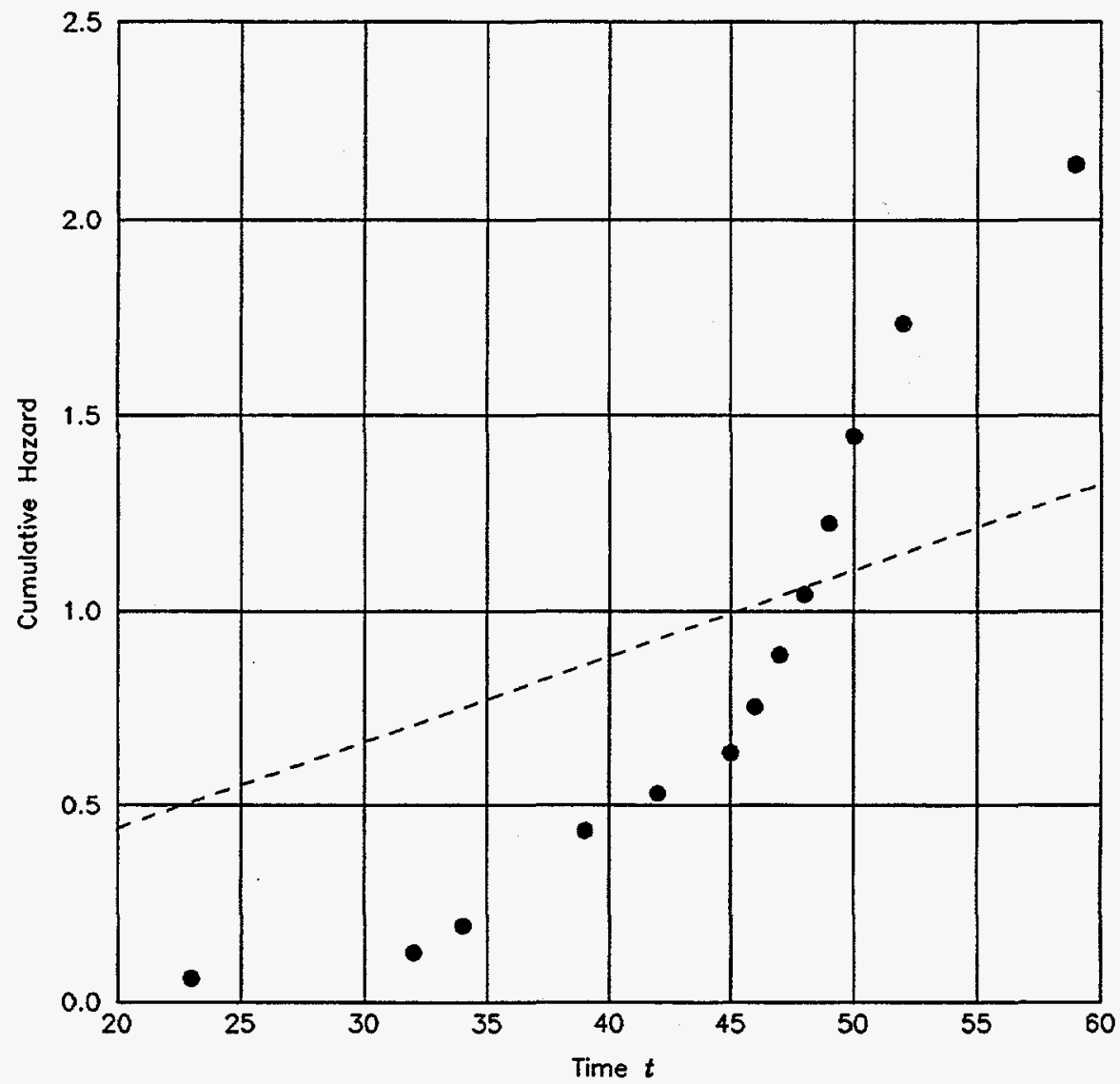

Figure 7. Cumulative hazard plot for outage-time data using linear scales.

dashed line in Figure 7 is the graph of the linear equation $y=\hat{\lambda} t$ with ML estimate $\hat{\lambda}=1 / \bar{t}=0.022$. Clearly, this plot does not support an assumption of exponential refueling times because the plotted points do not seem to be closely fitted by any line, much less the ML line which is superimposed on the graph. 
The CVM test can be performed for the exponential model with $\theta=\lambda$ and the data of Example 1. As noted, the ML of $\lambda$ is $\hat{\lambda}=0.022$, which can be used to compute $C M$. Table 10 of Bain and Engelhardt (1992, p.613) can be used to find the 0.99th quantile $C M_{0.99}=0.33<0.98=C M$, which leads to a rejection of the exponential model, consistent with previous results.

There are two important generalizations of the exponential distribution which provide more flexible models. These are the Weibull distribution and the gamma distribution.

\section{The Weibull Distribution}

Another often used distribution with random times is the Weibull distribution. A random variable $T$ has the Weibull distribution with parameters $\beta>0$ and $\theta>0$ if its pdf has the form

$f(t)=(\beta / \theta)(t / \theta)^{\beta-1} \exp \left[-(t / \theta)^{\beta}\right]$

for $t>0$, and zero otherwise. The parameter $\beta$ is called a shape parameter, and as with the Exponential model, $\theta$ is a scale parameter. The scale parameter $\theta$ has the same units as the random time $T$ and the shape parameter $\beta$ is unitless.

The Weibull model is a special case of the exponential model when $\beta=1$. Different values of $\beta$ lead to different shapes for the pdf and the hazard function. Figure 8 shows several Weibull pdfs for some different shape parameter values $\beta$, but with the same value of $\theta$.

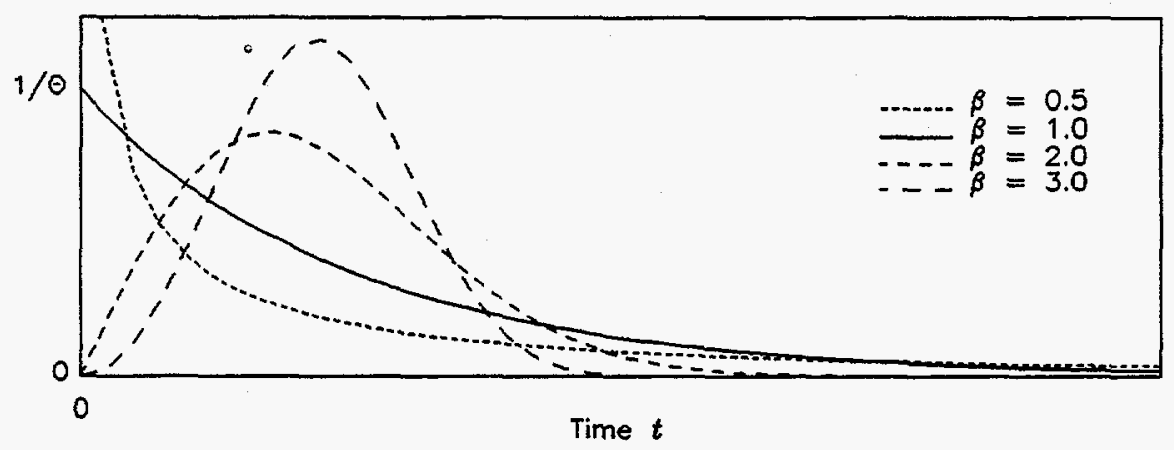

Figure 8. Weibull probability density functions for different shape parameter values $\beta$.

The CDF and reliability functions of the Weibull distribution are 
$F(t)=1-\exp \left[-(t / \theta)^{\beta}\right]$

and

$R(t)=\exp \left[-(t / \theta)^{\hat{\theta}}\right]$

It is clear, from the relationship between the reliability and cumulative hazard function noted previously, that

$H(t)=(t / \theta)^{\beta}$

and thus the hazard function, obtained by differentiating $H(t)$, is

$h(t)=(\beta / \theta)(t / \theta)^{\beta-1}$.

As noted previously, when $\beta=1$, the distribution is exponential. Also note that when $\beta<1$, the pdf is very concentrated near the origin and $f(t) \rightarrow \infty$ as $t \rightarrow 0$. Furthermore, the pdf is a decreasing function of $t$. On the other hand, when $\beta>1, f(0)=0$ and the pdf attains a unique mode for some $t$ $>0$. Figure 9 shows hazard functions for the same set of $\beta$-values used in Figure 8 . The hazard

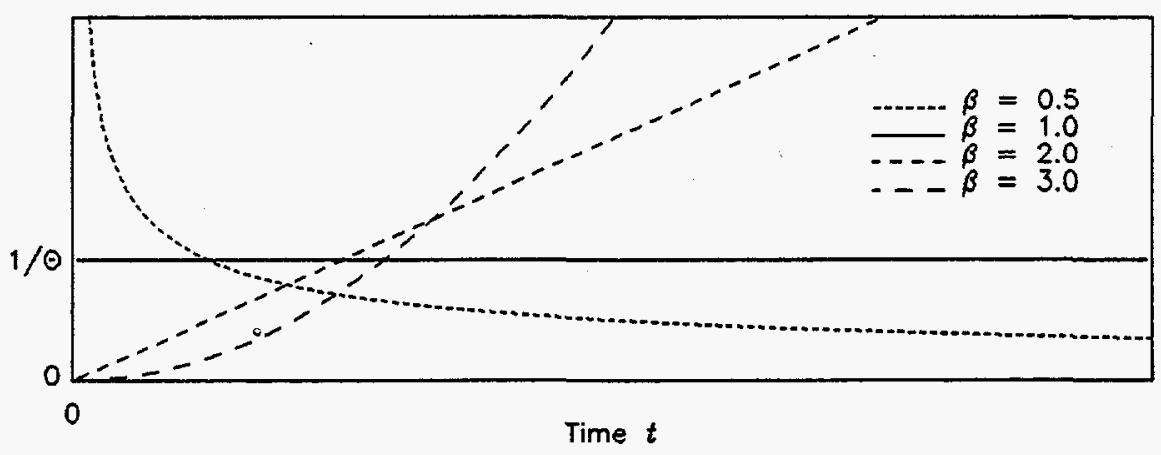

Figure 9. Weibull hazard functions for different shape parameter values $\beta$.

functions are increasing when $\beta>1$, decreasing when $\beta<1$, and constant in the exponential case $(\beta$ $=1$ ). In all decreasing cases, $h(t) \rightarrow \infty$ as $t \rightarrow 0$ and $h(t) \rightarrow 0$ as $t \rightarrow \infty$. In all increasing cases, $h(0)$ $=0$, and $h(t) \rightarrow \infty$ as $t \rightarrow \infty$. Therefore, Weibull distributions are capable of modeling fairly extensive wearout for aging units when $\beta>1$, as well as no wearout when $\beta=1$. Another interesting special case is when $\beta=2$. In this case, the Weibull distribution is called the Rayleigh distribution, and the 
hazard function is linear with slope $2 / \theta^{2}$ and zero intercept.

An important application of the Weibull distribution involves situations when the failure time of $a$ unit is the minimum of a large number $k$ of independent components. A system of this type is called a series system. For example, a chain which is stretched fails when its weakest link fails. For this reason, the Weibull distribution is often called the weakest link model. More generally, a series system fails at the first failure of one of its components.

More precisely, for a unit with $k$ independent components, if $T_{\mathrm{i}}$ is the failure of $i$ th component, then the time to failure of the unit is $T=\min \left(T_{1}, T_{2}, \ldots, T_{k}\right)$. Under certain conditions, this minimum has an approximate Weibull distribution. In particular, if the $T_{\mathrm{i}} \mathrm{s}$ are independent and identically distributed and 0 is the largest number $\tau$ such that $P\left[T_{\mathrm{i}}>\tau\right]=1$, (i. e. 0 is the greatest lower bound) then $T$ is approximately Weibull for large $k$. For more details of this property see Bain and Engelhardt (1992, p. 258). The Weibull distribution has been used widely in applications involving reliability and breaking strength of materials.

Even if the greatest lower bound $\tau$ is positive $(\tau>0)$, it can be shown that the related variable $T-\tau$ is approximately Weibull. When $T-\tau$ is Weibull distributed for some constant $\tau>0$, the value $\tau$ is called a threshold parameter, and $T$ is said to have a three-parameter Weibull distribution. A threshold parameter is appropriate if there is some initial period during which the probability of failure is zero. When $\tau=0$, we have the two-parameter Weibull distribution, or simply the Weibull distribution as defined above.

The mean and variance of a Weibull distribution with parameters $\beta$ and $\lambda$ are

$E(T)=\theta \Gamma(1+1 / \beta)$

$\operatorname{var}(T)=\theta^{2}\left[\Gamma(1+2 / \beta)-\Gamma^{2}(1+1 / \beta)\right]$

As noted above, the cumulative hazard function is of the form $H(t)=(t / \theta)^{\beta}$. If we apply the logarithm function we obtain

$\log [H(t)]=\beta \log (t)-\beta \log (\theta)$

which is a linear function of $\log (t)$ with slope $\beta$ and $y$-intercept $-\beta \log (\theta)$. This suggests plotting $\log [H(t)]$ versus $\log (t)$ and inspecting it for an approximately linear pattern. Another alternative would be to plot $H(t)$ versus $t$ on log-log paper (i.e. with a log-scale both on the horizontal and vertical axes). 
This last situation is shown in Figure 10 with the data from Example 1. This shows a much better fit

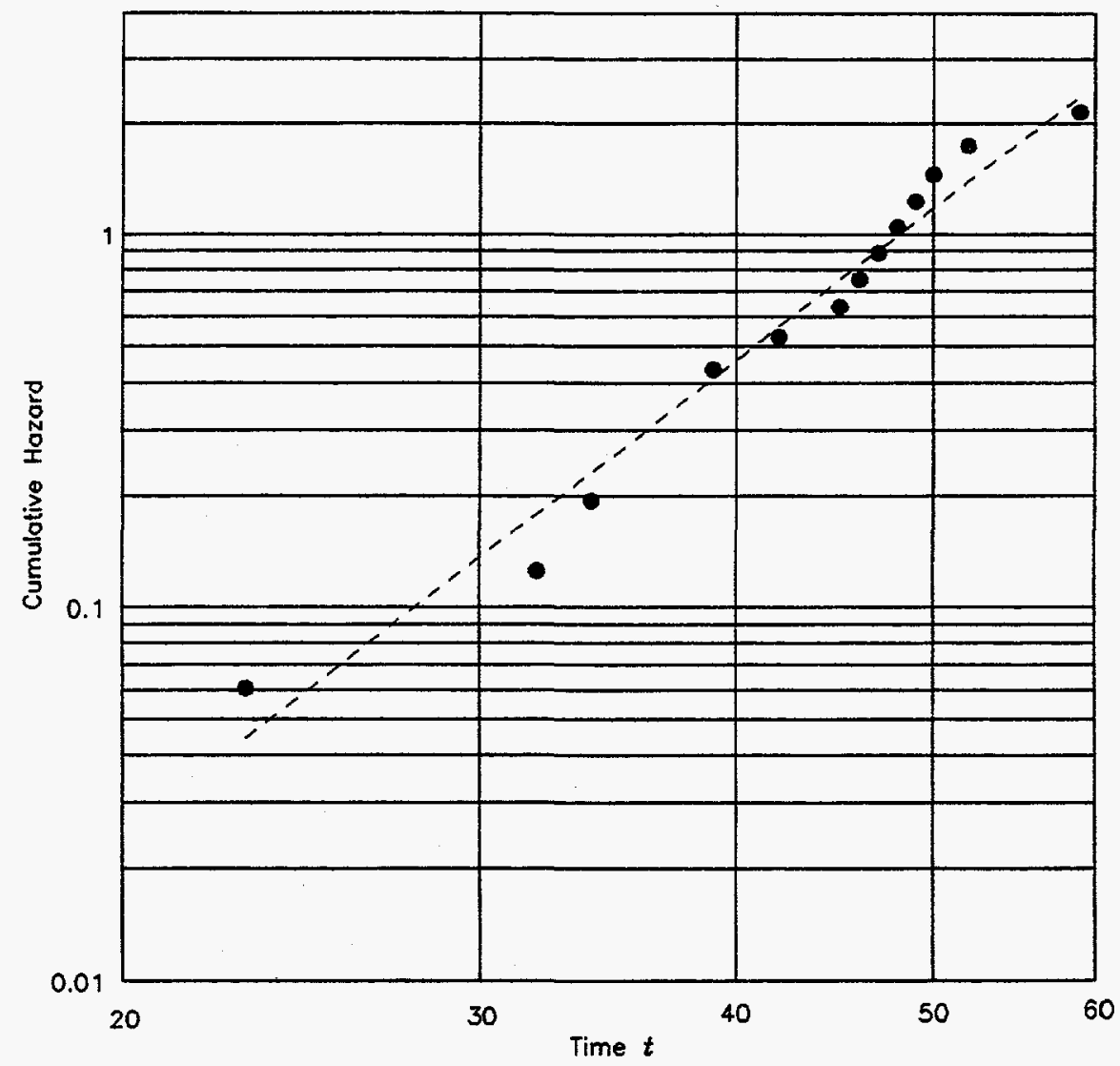

Figure 10. Cumulative hazard plot of refueling times with log-log scale.

to the data than we saw in Figure 7 which involved the exponential distribution. It is possible to obtain graphical estimates of the Weibull parameters from such a plot. In particular, the slope of a fitted line in log-log scale provides an estimate of the shape parameter and the $y$-intercept provides an estimate of the product $-\beta \log (\theta)$. The linear least squares estimates of slope and intercept for $y=\log \left[\hat{H}\left(t_{i}\right)\right]$ versus $x=\log \left(t_{\mathrm{i}}\right)$ are slope estimate $=\hat{\beta}=4.21$ and intercept estimate $=-\hat{\beta} \log (\hat{\theta})=-16.32$. The estimate for $\theta$, using the least squares estimates, is $\hat{\theta}=\exp (16.32 / 4.21)=48.3$.

It would be desirable to get formal confirmation of the Weibull fit by means of a goodness-of-fit 
test. The CVM test can be performed using ML estimation with $\beta$ and $\theta$. Although it is possible to compute the ML estimates and confidence intervals for the parameters of a Weibull distribution, the computations are somewhat harder than they were with an exponential distribution. The ML estimates of $\beta$ and $\theta$ are solutions $\hat{\beta}$ and $\hat{\theta}$ to the pair of equations

$$
\frac{\sum_{i=1}^{n} t_{i}^{\beta} \log \left(t_{i}\right)}{\sum_{i=1}^{n} t_{i}^{\beta}}-\frac{1}{\beta}-\frac{\sum_{i=1}^{n} \log \left(t_{i}\right)}{n}=0
$$

and

$$
\hat{\theta}=\left(\frac{1}{n} \sum_{i=1}^{n} t_{i}^{\beta}\right)^{1 / \hat{\beta}}
$$

The first of these equations cannot be solved explicitly as a function of the data, but for a given set of numerical data it is possible to solve for $\hat{\beta}$ by an iterative numerical method such as the Newton-Raphson method. Once this equation is solved, the ML estimate of $\beta$ can be substituted into the second equation, yielding the ML estimate of $\theta$. The SAS procedure PROC LIFEREG can be used to compute the ML estimates. For the data of Example 1, the ML estimates are $\hat{\beta}=4.90$ and $\hat{\theta}=49.4$.

Using these ML estimates, the value of the statistic is $C M=0.038$ and from Table 10 of Bain and Engelhardt (1992, p. 613), we can compute the 0.90 quantile as $C M_{0.90}=0.097>C M$, so the Weibull model cannot be rejected at the 0.10 level of significance.

Based on the ML estimates of $\beta$ and $\theta$ we can also compute the ML estimates of $R(t), \mu=E(T)$ and $\sigma^{2}=\operatorname{var}(T)$. These are obtained by substituting $\hat{\beta}$ and $\hat{\theta}$ into the formulas for the quantities $R(t)$, $\mu=E(T)$ and $\sigma^{2}=\operatorname{var}(T)$. For example, the ML estimate of $R(35)$ is $\hat{R}(35)=\exp \left[-(35 / 49.4)^{4.90}\right]=$ 0.84 . This is fairly close to the nonparametric estimates of 0.82 and 0.83 obtained using, respectively, the ERF and the Nelson estimate of reliability. The estimates of the mean time of refueling outage $\mu$ and the standard deviation $\sigma$ are $\hat{\mu}=(49.4) \Gamma(1+1 / 4.90)=45.9$ days, and $\hat{\sigma}=(49.4)[\Gamma(1+2 / 4.90)-$ $\left.\Gamma^{2}(1+1 / 4.90)\right]^{1 / 2}=10.7$ days. Recall that the sample mean and sample standard deviation, which are nonparametric estimates of the population mean and standard deviation, were 45.3 and 10.7 days.

Confidence intervals for the parameters $\beta$ and $\theta$, based on the ML estimates can be found, but special tables are required. These tables are given by Bain and Engelhardt (1991, Ch. 4). The confidence limits for a $(1-2 \alpha) 100 \%$ confidence interval for $\beta$ are of the form 
$\beta_{\mathrm{L}}=\hat{\beta} /\left(1+n^{-1 / 2} b_{1-\alpha}\right)$

and

$\beta_{U}=\hat{\beta} /\left(1+n^{-1 / 2} b_{\alpha}\right)$

where $b_{I-\alpha}$ and $b_{\alpha}$ are constants. Interpolation in Table 3A of Bain and Engelhardt $(1991$, p. 224) with $n=17$ and $\alpha=0.05$ yields the constants $b_{0.05}=-0.908$ and $b_{0.95}=2.037$. The limits for a $90 \%$ confidence interval for $\beta$ are 3.28 and 6.28 .

A $(1-2 \alpha) 100 \%$ confidence interval for the scale parameter $\theta$, based on the ML estimates, can be computed using results in Bain and Engelhardt (1991, p.228). These limits are of the form

$\theta_{\mathrm{L}}=\hat{\theta} \exp \left(-n^{-1 / 2} u_{1-\alpha} / \hat{\beta}\right)$

and

$\theta_{\mathrm{U}}=\hat{\theta} \exp \left(-n^{-1 / 2} u_{\alpha} / \hat{\beta}\right)$

where $u_{\alpha}$ and $u_{1-\alpha}$ are constants which are tabulated by Bain and Engelhardt (1991, Table 4A, p. 230). Interpolation in Table $4 \mathrm{~A}$ of with $n=17$ and $\alpha=0.05$ yields the constants $u_{0.05}=-1.943$ and $u_{0.95}=$ 1.908. The limits for a $90 \%$ confidence interval for $\theta$ are 45.5 and 55.6 .

Lower confidence limits for $R(t)$, using ML estimates, can also be found using tables from Bain and Engelhardt (1991, Table 7A). Using interpolation in this table, we obtain as a lower $95 \%$ confidence limit $R_{\mathrm{L}}(35)=0.68$. This also yields an upper $95 \%$ confidence limit of 0.32 for the probability that a randomly selected refueling outage time will not exceed 35 days. This upper limit is somewhat tighter than the nonparametric $95 \%$ upper confidence limit 0.40 obtained using the binomial distribution.

Of course, one advantage with using parametric methods rather than nonparametric methods is an improvement in efficiency. For point estimates, improved efficiency means a smaller standard error (less scatter) in computed point estimates, and for interval estimates improved efficiency translates into tighter bounds and shorter confidence intervals. As noted previously, when analyzing reliability, lower limits are generally considered to be more important than upper limits, and tables for computing upper confidence limits are not readily available. Presumably, if such an upper limit could be computed, the resulting confidence interval would be shorter than the nonparametric interval given previously. 


\section{The Gamma Distribution}

A random variable $T$ is gamma distributed with parameters $\kappa>0$ and $\theta>0$ if the pdf is

$f(t)=(1 / \theta)^{\kappa} t^{\kappa-1} \exp (-t / \theta) / \Gamma(k)$

for $t>0$, and zero otherwise where $\Gamma(\cdot)$ is the gamma function. The parameter $\kappa$ is called a shape parameter, and $\theta$ is a scale parameter. The scale parameter $\theta$ has the same units as the random time $T$. The shape parameter $\kappa$ is unitless. The notation $\beta=1 / \theta$ is often used in probabilistic risk assessment applications.

The exponential model is a special case of the gamma model when $\kappa=1$. Different values of $\kappa$ lead to different shapes for the pdf and the hazard function, which helps explain the term shape parameter. Figure 11 shows gamma pdfs for some different shape parameter values $\kappa$, but the same $\theta$.

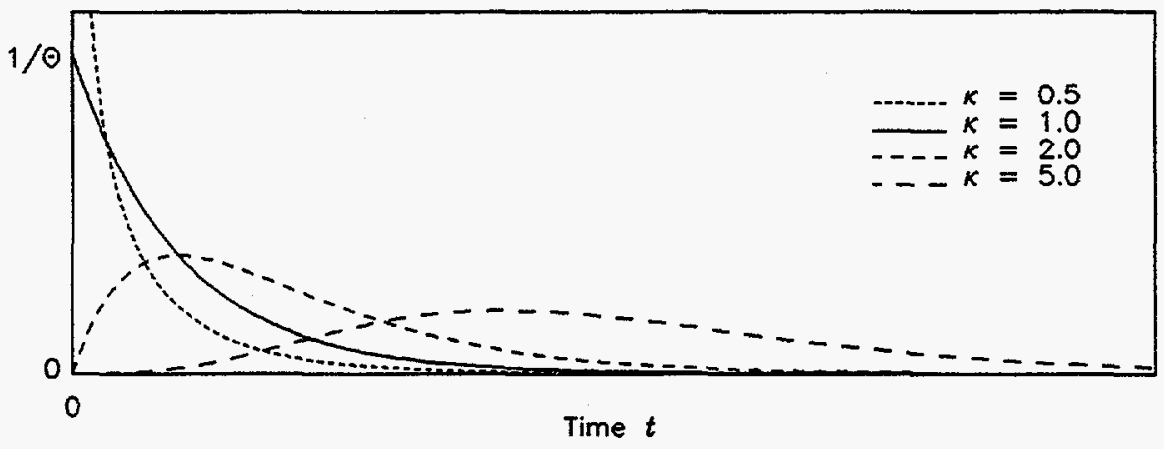

Figure 11. Gamma probability density functions for different shape parameter values $\kappa$.

As noted previously, when $\kappa=1$ the distribution is exponential. Also note that when $\kappa<1$ the pdf is very concentrated near the origin and $f(t) \rightarrow \infty$ as $t \rightarrow 0$. Furthermore, the pdf is a decreasing function of $t$. On the other hand, when $\kappa>1, f(0)=0$ and the pdf has a unique mode for some $t>$ 0 . Figure 12 shows hazard functions for the same set of $\kappa$ values used in Figure 11. It is interesting to note that the hazard functions are increasing when $\kappa>1$, decreasing when $\kappa<1$, and of course constant in the exponential case $(\kappa=1)$. In all cases the hazard function approaches the limiting value $1 / \theta$. It can be shown that in general for a gamma distribution with parameter $k$ and $\lambda$, the hazard function is increasing when $\kappa>1$, decreasing when $\kappa<1$, and regardless of the value of $\kappa$, the hazard function approaches $1 / \theta$ as $t \rightarrow \infty$. Therefore, gamma distributions are capable of modeling a moderate amount 


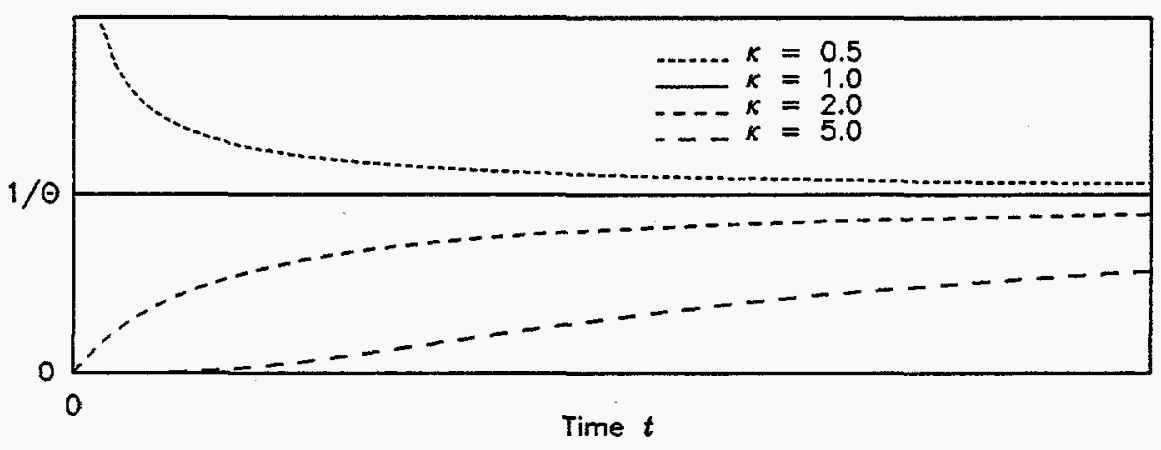

Figure 12. Gamma hazard functions for different shape parameter values $\kappa$.

of wearout for aging units when $\kappa>1$, as well as no wearout when $\kappa=1$.

An important situation involves units which are known to fail when subjected to a fixed number of "shocks" $k$ (and not sooner), where the times between shocks are independent and exponential with scale parameter $\theta$. If $T_{i}$ is the time between shock $i-1$ and shock $i$, then the time to failure of the unit is $T=T_{1}+T_{2}+\cdots+T_{k}$. This sum can be shown to be gamma distributed with parameters $\kappa=$ $k$ and $\theta$. The gamma distribution is called the Erlang distribution when the shape parameter is a positive integer, as in this situation.

Explicit expressions for the CDF, reliability and hazard functions can be derived when the shape parameter is an integer $k$. In particular, the integral which defines the reliability can be integrated by parts repeatedly to express it as the following sum:

$$
R(t)=\int_{t}^{\infty} \frac{1}{\theta(k-1) !}(u / \theta)^{k-1} \exp (-u / \theta) d u=\sum_{i=0}^{k-1} \frac{(t / \theta)^{i}}{i !} \exp (-t / \theta)
$$

The corresponding hazard function is

$$
h(t)=\frac{f(t)}{R(t)}=\frac{(1 / \theta)(t / \theta)^{k-1} /(k-1) !}{\sum_{i=0}^{k-1}(t / \theta)^{i} / i !}
$$

It is difficult, for general $\kappa>0$ to give explicit expressions for the reliability function of a gamma distribution, but they can be expressed in terms of the $\chi^{2}$ distribution whose tabled values are widely available. In particular, if $T$ is gamma with parameters $\kappa$ and $\theta$, and if $G(; \nu)$ is the CDF of the $\chi^{2}$ 
distribution with $\nu$ degrees of freedom, then

$R(t)=1-G(2 t / \theta ; 2 \kappa)$

The mean and variance of a gamma distribution with parameters $\kappa$ and $\lambda$ are

$E(T)=\kappa \theta$

$\operatorname{var}(T)=\kappa \theta^{2}$.

The ML estimate for $E(T)$ is the sample mean, $\bar{t}$, but the ML estimates for the individual parameters in a bit more complicated. These ML estimates are given by Bain and Engelhardt (1991, p. 297) with the scale parameter $\theta$. The ML estimates are solutions of the equations

$\hat{\theta}=\bar{t} / \hat{\kappa}$

and

$\log (\hat{\kappa})-\psi(\hat{\kappa})=\log (\bar{t} / \tilde{t})$

where $\psi(z)=\Gamma^{\prime}(z) / \Gamma(z)$ is the digamma function and $\tilde{t}=\left[\Pi t_{i}\right]^{1 / n}$ is the geometric mean of the observed times $t_{\mathrm{i}}$. The second equation can be solved by iterative numerical methods. Because $\hat{\kappa}$ is a function only of $\bar{t} / \tilde{t}$ and does not depend separately on $n, \bar{t}$ and $\tilde{t}$ it is rather easily tabled. Such a table is given by Bain and Engelhardt (1991, p. 298). For the data of Example 1, the MLEs are $\hat{\kappa}=17.42$ and $\hat{\theta}=2.63$. Methods for computing confidence limits for the parameters $\kappa, \theta$ and $E(T)$ are also discussed by Bain and Engelhardt (1991), but we will not discuss those results here.

Result on the CVM test for the gamma model with unknown parameters are discussed by D'Agostino and Stephens (1986, pages 151-156). The value of the statistic is $C M=0.035$. Based on Table 4.21 of D'Agostino and Stephens, this has a $p$-value greater than 0.25 . Thus, the gamma distribution would also be a reasonable model for these data.

The Normal Distribution

A random variable $T$ has the normal distribution with mean $\mu$ and variance $\sigma^{2}$ if it has pdf 


$$
f(t)=\frac{1}{\sqrt{2 \pi \sigma^{2}}} \exp \left(-\frac{(t-\mu)^{2}}{2 \sigma^{2}}\right)
$$

The special case with $\mu=0$ and $\sigma^{2}=1$ is the standard normal distribution with pdf

$$
\phi(t)=\frac{1}{\sqrt{2 \pi}} \exp \left(-t^{2} / 2\right)
$$

and CDF denoted by $\Phi(t)$. For an arbitrary normal random variable $T$ with mean $\mu$ and variance $\sigma^{2}$, the CDF, which can be evaluated from tables, or obtained by numerical integration of the pdf $\phi$, is

$$
F(t)=\Phi\left(\frac{t-\mu}{\sigma}\right)
$$

The normal distribution is sometimes used for a lifetime distribution, even though it allows negative values of $T$ with positive probability. Thus, strictly speaking it shouldn't be used as a lifetime model unless $\mu$ is enough larger than $\sigma$ that the chance of observing a negative value is negligible, say $\mu>3 \sigma$. The hazard function of the standard normal distribution is

$$
h_{0}(t)=\frac{\phi(t)}{1-\Phi(t)}
$$

and for an a normal distribution with mean $\mu$ and variance $\sigma^{2}$, the hazard function is

$$
h(t)=\frac{1}{\sigma} h_{0}\left(\frac{t-\mu}{\sigma}\right)
$$

The hazard function for an arbitrary normal distribution differs from that of a standard normal by a change of location and scale, similar to the relationship for pdfs. The function $h_{0}(t)$ is known to be increasing and its graph, $y=h_{0}(t)$ approaches the line $y=t$ asymptotically as $t \rightarrow \infty$. The standard normal pdf, CDF and hazard function are shown is Figure 13.

It is possible to do hazard plotting for the normal model, but it requires plotting paper with a specially constructed vertical scale. Such paper is not always easy to find. Thus, we will rely only on goodness-of-fit testing to check the model. The same problems discussed above for applying the chisquare goodness-of-fit test with small sets of data and a two-parameter model apply with the normal model. 


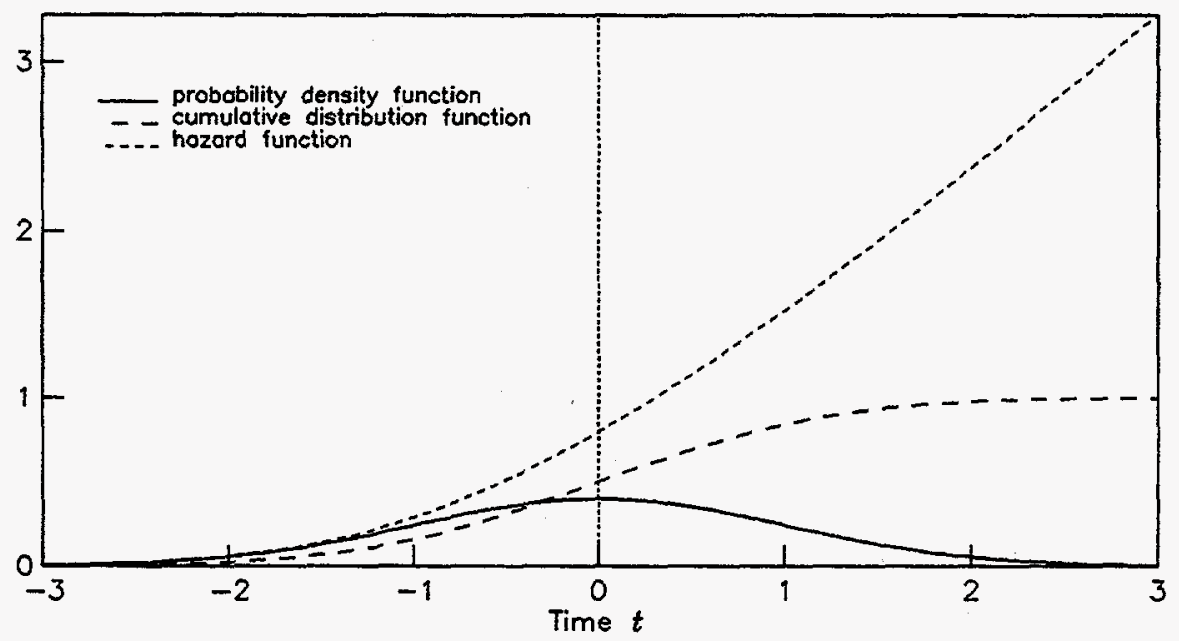

Figure 13. Standard normal probability density, cumulative distribution and hazard functions.

An easy way to test for fit with the normal model is to use the CVM test. For the normal model, and again considering Example 1, the ML estimates are $\hat{\mu}=\bar{t}=45.29$ and $\hat{\sigma}^{2}=(1 / n) \Sigma\left(t_{\mathrm{i}}-\bar{t}\right)^{2}=$ 108.09. The computed CVM statistic is $C M=0.029$, and the 0.90 th quantile for the CVM statistic, from Table 10 of Bain and Engelhardt is $C M_{0.90}=0.101>0.029$, which indicates that the normal distribution cannot be rejected.

As noted previously, the $\mathrm{ML}$ estimate of $E(T)$ is $\bar{t}=45.29$, and the $\mathrm{ML}$ estimate of the population standard deviation $\sigma$ is 10.40 . The ML estimate of $R(35)$ is $1-\Phi((35-45.29) / 10.40)=$ $\Phi(0.99)=0.84$. Confidence limits can be computed for $\mu, \sigma^{2}$ and $R(t)$. Limits for a $(1-2 \alpha) 100 \%$ confidence interval for $\mu$ are

$\mu_{\mathrm{L}}=\bar{t}-t_{1-\alpha, n-1} s / n^{1 / 2}$

and

$\mu_{\mathrm{U}}=\bar{t}+t_{1-\alpha, n-1} s / n^{1 / 2}$

where $s$ is the sample standard deviation and $t_{1-\alpha, n-1}$ is the $(1-\alpha)$ th quantile of the $t$ distribution with $n-1$ degrees of freedom. Limits for a $(1-2 \alpha) 100 \%$ confidence interval for $\sigma^{2}$ are of the form 
$\sigma_{L}^{2}=(n-1) s^{2} / \chi_{1-\alpha, n-1}^{2}$

and

$\sigma_{\mathrm{U}}^{2}=(n-1) s^{2} / \chi_{\alpha, n-1}^{2}$

Using these formulas, we obtain a $90 \%$ confidence interval $(40.8,49.8)$ for $\mu$, and a $90 \%$ confidence interval $(69.9,230.9)$ for $\sigma^{2}$. By taking square root of the limits in the latter interval, we obtain confidence limits $(8.4,15.2)$ for $\sigma$.

A procedure for computing an approximate lower confidence limit for $R(t)$ is given by Bain and Engelhardt (1991, p. 376). Specifically,

$$
R_{\mathrm{L}}(t) \approx \Phi^{-1}\left(\frac{\bar{t}-t}{s}-z_{1-\epsilon}\left[\frac{1}{n}+\frac{(\bar{t}-t)^{2} / s^{2}}{2(n-1)}\right]^{1 / 2}\right)
$$

where $\Phi^{-1}$ is the inverse of the standard normal CDF. In our application, we get $R_{\mathrm{L}}(35) \approx \Phi^{-1}(0.47)$ $=0.68$. Also, $1-0.68=0.32$ is a $95 \%$ upper confidence limit on $F(35)$.

Another question which often comes up, when analyzing data, is whether two sets of data from similar sources can be combined. For example, we have been treating the combined data from two plants at the same station as data from one source. It is well known that mixing data from two or more dissimilar populations can lead to incorrect inferences (see e.g. Atwood, 1995, p. 5). Consequently, it is of interest to consider tests which test for differences in the underlying populations. With data from normal populations such tests are readily available.

A test of equality of means $\mathrm{H}_{0}: \mu_{1}=\mu_{2}$ versus $\mathrm{H}_{2}: \mu_{1} \neq \mu_{2}$ is available based on a t-statistic. If we assume equality of population variances $\sigma_{1}^{2}=\sigma_{2}^{2}$, then a size $\alpha$ test of $\mathrm{H}_{0}$ rejects if $|t| \geq t_{1-\alpha / 2, \nu}$ where $t=\left(\bar{t}_{1}-\bar{t}_{2}\right) /\left[s_{p}\left(1 / n_{1}+1 / n_{2}\right)^{1 / 2}\right]$ with $\left.s_{\mathrm{p}}^{2}=\left[\left(n_{1}-1\right) s_{1}^{2}+\left(n_{2}-1\right) s_{2}^{2}\right)\right] / \nu$ and $\nu=n_{1}+n_{2}-2$. A modification of the t-test which holds when $\sigma_{1}^{2} \neq \sigma_{2}^{2}$ and a test for equality of variances are discussed by Bain and Engelhardt (1992, p. 402-403). As an example, consider the data from Plants A and B in Example 1. For Plant A, $n_{1}=9, \bar{t}_{1}=42.22$ and $s_{1}=10.33$, while for Plant B $n_{2}=8, \bar{t}_{2}=48.75$ and $s_{2}=10.71$. Assuming a common value of $\sigma$, then pooled estimate is $s_{p}=10.51$, and the t-statistic is $t=1.28$ with $\nu=9+8-2=15$ degrees of freedom. The $p$-value of this test is 0.11 , which is not strong evidence that the means are different. 


\section{The Lognormal Distribution}

Another distribution related to the normal distribution is obtained if a logarithmic transformation yields normally distributed data. In particular, $T$ is said to have a lognormal distribution with parameters $\gamma$ and $\delta$ if $Y=\log (T)$ is normally distributed with mean $\gamma$ and variance $\delta^{2}$. Because of this relationship to the normal distribution, we can write the pdf and CDF as

$$
f(t)=\frac{1}{\delta t} \Phi\left(\frac{\log (t)-\gamma}{\delta}\right)
$$

and

$$
F(t)=\Phi\left(\frac{\log (t)-\gamma}{\delta}\right)
$$

for $t>0$ and zero otherwise. The pdfs for several pairs of parameters $\gamma$ and $\delta$ are shown in Figure 14 .

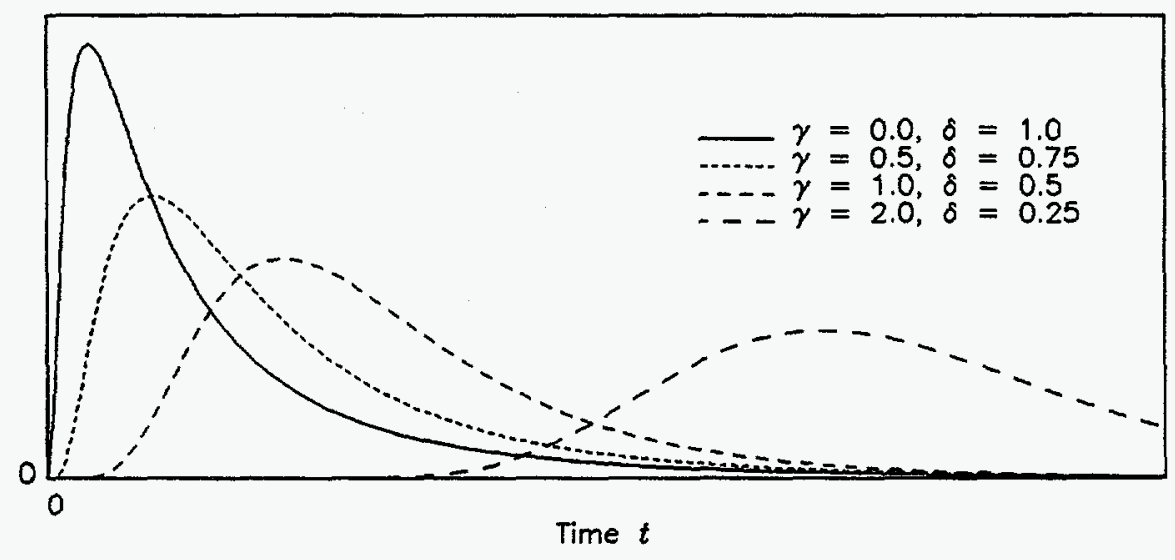

Figure 14. Lognormal probability density functions for different combinations of $\gamma$ and $\delta$.

It is clear that the lognormal distribution is capable of modeling skewed distribution similar to the gamma and Weibull model. However, the hazard functions behave somewhat differently, as seen in Figure 15. Hazard functions for lognormal distributions are "hump" shaped. That is, they attain the values zero at $t=0, h(0)=0$, and are increasing until they attain a maximum at some value $t>0$, after which they decrease to zero, $h(t) \rightarrow 0$ as $t \rightarrow \infty$. It is not obvious how to interpret a hump shaped hazard function for a lifetime model. However, the lognormal distribution is a popular choice in studies of lifetime. A general discussion of properties of lognormal hazard function is given by Sweet (1990). 


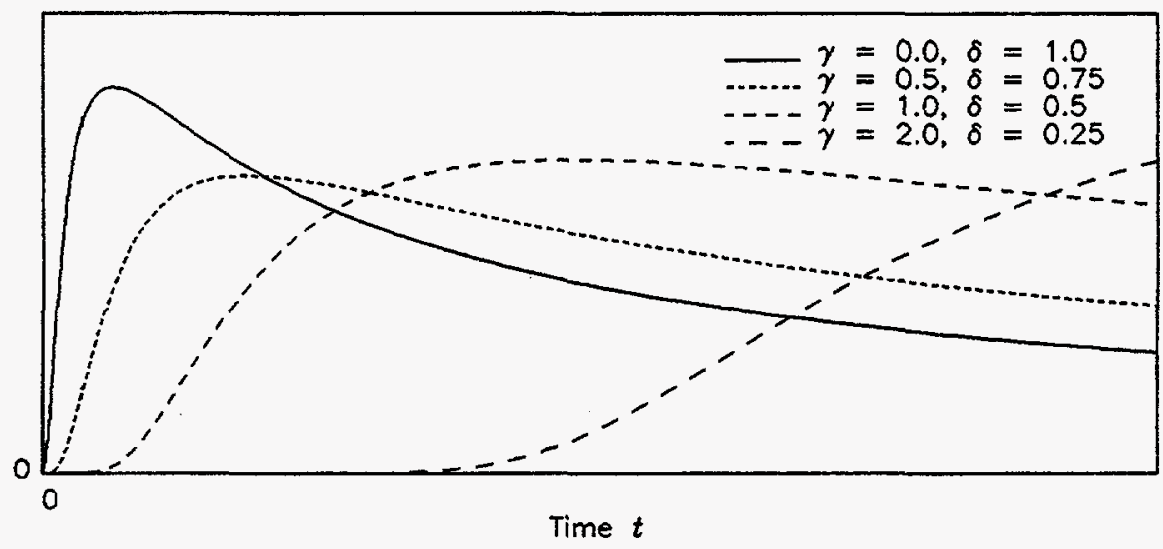

Figure 15. Hazard function of the lognormal distribution for different combinations of $\gamma$ and $\delta$.

The mean and variance for a lognormal distribution with parameters $\gamma$ and $\delta$ are

$\mathrm{E}(T)=\exp \left(\gamma+\delta^{2} / 2\right)$

and

$\operatorname{var}(T)=\exp (2 \gamma)\left[\exp \left(2 \delta^{2}\right)-\exp \left(\delta^{2}\right)\right]$

The $100 p$ th percentile of a lognormal distribution is

$t_{p}=\exp \left(\gamma+\delta z_{p}\right)$

where $z_{p}$ is the $100 p$ th percentile of the standard normal distribution. Due to the symmetry of the normal distribution about its mean, the median of a lognormal distribution is simply

$t_{\text {med }}=\exp (\gamma)$

If $p<0.5$, upper and lower limits which hold with a specified probability $(1-2 p)$ for a lognormal random variable $T$ are defined by

$t_{\mathrm{U}}=\exp \left(\gamma+\delta z_{1-p}\right)=t_{\text {med }} k$ 
and

$t_{\mathrm{L}}=\exp \left(\gamma-\delta z_{1-p}\right)=t_{\text {med }} / k$

where

$k=\exp \left(\delta z_{1-p}\right)$

It follows that

$\mathrm{P}\left[t_{\text {med }} / k<T<t_{\text {med }} k\right]=1-2 p$

The constant $k$ is called the $(1-2 p)$ error factor. For example, if $p=0.05$, then $k$ is the 0.90 error factor, and $90 \%$ of all units with this life distribution will have lifetimes between these limits. The median is a measure of central tendency and the error factor provides a measure of dispersion with a larger value of $k$ corresponding to greater dispersion.

An interesting application of the error factor is found in the reactor safety study WASH-1400 (1975). It was assumed that the failure rate $\lambda$ was constant for any given power plant, but that the failure rates vary from plant to plant with variation modeled by a lognormal distribution. The uncertainty in $\lambda$ was expressed by an interval $\left(\lambda_{L}, \lambda_{U}\right)$ such that $\mathrm{P}\left[\lambda_{\mathrm{L}}<\lambda<\lambda_{\mathrm{U}}\right]=0.90$. Because a lognormal distribution is assumed, the uncertainty can be expressed in terms of the median failure rate, say $\lambda_{\text {med }}=$ $\exp (\gamma)$, and a $90 \%$ error factor $k=\exp (1.645 \delta)$.

Lognormal hazard plots are possible, but it requires plotting paper with a specially constructed vertical scale and a logarithmic horizontal scale. This will not be considered here.

An easy way to test for fit is with the CVM test. The CVM test can be used to test the lognormal distribution after a logarithmic transformation of the data. That is, the CVM test of normality can be applied to $\log \left(t_{j}\right)$ values. The ML estimates are $\hat{\gamma}=(1 / n) \Sigma \log \left(t_{i}\right)=3.78$ and $\hat{\delta}=\left\{(1 / n) \Sigma\left[\log \left(t_{i}\right)\right]^{2}-\right.$ $\left.\hat{\gamma}^{2}\right\}^{1 / 2}=0.248$, and the CVM statistic is $C M=0.045$ and the 0.90 th quantile for the CVM statistic, from Table 10 of Bain and Engelhardt (1992) as before is $C M_{0.90}=0.101>0.045$, which indicates that the lognormal distribution also cannot be rejected.

It should be noted at this point that the exponential model is the only one considered so far which clearly gives a bad fit to the data of Example 1. The CVM tests for the Weibull, normal and lognormal all had $p$-values greater than 0.10 . The choice among these nonrejected models is unclear. One possible 
idea would be to choose the model whose CVM test has the largest $p$-value. A practical problem with this is that the tabled quantiles can't be interpolated to find $p$-values larger than 0.10 .

Another, more serious question, is whether failure to reject a model means that this is a "correct" model. The answer is that failure to reject a model does not prove that a model is true. In other words, goodness-of-fit test, such as the CVM test, is designed to guard against rejecting a model that fits well, and if different models are tested for goodness of fit, more than one model may be accepted unless the data set it fairly large. The data set for Example 1 is fairly small, and the fact that different models are accepted suggests that the data set is not large enough to show conclusively that any of the nonrejected models is more appropriate than any other.

The analyses applied under the normal assumption could be carried out to obtain confidence intervals for the mean and variance of the distribution of the $\log \left(T_{i}\right) \mathrm{s}$, but this will not provide confidence intervals for the mean and variance of the original lognormal population. It is possible to adapt the results for the reliability from normal to lognormal. In particular, the ML estimate of $R(t)$ is

$$
\hat{R}(t)=\Phi\left(\frac{\hat{\gamma}-\log (t)}{\delta}\right)
$$

Based on the data from Example 1, we obtain $\hat{R}(35)=\Phi((3.78-\log (35)) / 0.248)=\Phi(0.906)=0.82$. Approximation (4) for a lower confidence limit on reliability can be used if we substitute $\log (t)$ for $t$, and replace $\bar{t}$ and $s$ in (4) with their counterparts based on the log-data. In other words, we replace $\bar{t}$ with $\hat{\gamma}$ and $s$ with $[n /(n-1)]^{1 / 2} \hat{\delta}$. Using the data of Example 1 , the $95 \%$ lower confidence limit is $R_{\mathrm{L}}(35) \approx$ $\Phi^{-1}(0.41)=0.66$. Thus, in this example, the $95 \%$ upper confidence limit for $F(35)$ is $1-0.66=0.34$, which is not quite as tight as the limits obtained under either the Weibull and normal assumptions. It would require a comparative study to know if this is true for other times $t$, or possibly to explain it. These models are quite different, and one should not expect the same results with different assumptions.

\section{Some Related Concepts}

It is possible to use PROC LIFEREG from the SAS software system to compute estimates of unknown parameters for some of the models discussed above. However, it is necessary to understand the relationship between some of the models for duration times and models obtained when the time variable is transformed with a logarithmic transformation. 


\section{Location-Scale Models}

In general, a probability distribution for a random variable $Y$ is said to be a location-scale model if it has a CDF of the form

$$
F(y ; a, b)=G\left(\frac{y-a}{b}\right)
$$

for real numbers $-\infty<a<\infty$ and $b>0$ where $G$ is a known function having the properties of a CDF and free of unknown parameters. In this setting, $a$ is called a location parameter and $b$ is called a scale parameter. We have already encountered one location-scale model, namely the normal distribution. Recall, the CDF for a normally distributed random variable $Y$ with mean $\mu$ and standard deviation $\sigma$ can be written as

$$
F(y ; \mu, \sigma)=\Phi\left(\frac{y-\mu}{\sigma}\right)
$$

so that the normal distribution is a location-scale model with location parameter $a=\mu$, scale parameter $b=\sigma$, and standard normal CDF $G(z)=\Phi(z)$.

It is also possible to have one-parameter models which are either purely location or purely scale models. For example, the distribution of a random variable $Y$ is a location model if its CDF is of the form

$$
F(y ; a)=G(y-a)
$$

and similarly, the distribution is a scale model if its CDF is of the form

$$
F(y ; b)=G\left(\frac{y}{b}\right)
$$

where $G$ is a known CDF free of unknown parameters. Notice that the exponential distribution with $b$ $=1 / \lambda$ is a scale model. The normal distribution with known standard deviation, say $\sigma=1$, but unknown mean is a location model.

Another important example of a location-scale model is called the Type I extreme-value distribution, which has CDF of the form

$$
F(y ; a, b)=1-\exp \left[-\exp \left(\frac{y-a}{b}\right)\right]
$$

for $-\infty<y<\infty$. 
Although, as noted previously, the gamma distribution is sometimes used as a model for duration times, there is no known transformation which relates a gamma distribution to a location-scale model. Consequently, none of the properties discussed here for location-scale models apply with the gamma distribution.

\section{Probability Plotting}

An important graphical method for choosing models can be used within the location-scale framework. In particular, a popular approach is called probability plotting. This amounts to plotting the inverse of $G$ at special plotting points $p_{\mathrm{i}}$ versus the observed values of the order statistics $y_{(i)}$ (see e.g. D'Agostino and Stephens, 1986, p. 34). Roughly, $p_{i}$ is approximately the probability that an observed value of $Y$ will not exceed $y_{(i)}$, or in other words, $p_{i} \approx F\left(y_{(i)} ; a, b\right)$. An often used choice for plotting position is $p_{i}=(i-0.5) / n$. Thus, a probability plot is a plot of $z_{i} \equiv G^{-1}\left(p_{i}\right)$ on the vertical axis versus $y_{(i)}$ on the horizontal axis. If a location-scale model is appropriate and the choice of $G$ is correct, then such a plot will be approximately linear. This follows from the approximate relationship

$G^{-1}\left(p_{i}\right) \approx\left(y_{(i)}-a\right) / b$

which can also be written as

$y_{(i)} \approx a+b z_{i}$

or, in other words, $y_{(i)}$ is approximately a linear function of $z_{i}$ with intercept $a$ and slope $b$. This is an alternative approach to hazard plotting which was discussed previously in connection with the models for duration times. We will not pursue the connection between probability plots and hazard plots further, but a discussion of this connection can be found in D'Agostino and Stephens (1986, p.469).

\section{Transformations}

It turns out that some distributions which are not location-scale models are related to locationscale models by a log-transformation of the random variable. One example which we have already seen is the lognormal distribution. That is, if $T$ is lognormally distributed its CDF is not a location-scale model, but $Y=\log (T)$ has a normal distribution which is a location-scale model with location parameter $a=\gamma$ and scale parameter $b=\delta$.

Another important example is the Weibull distribution. In particular, if $T$ is Weibull distributed with shape parameter $\beta$ and scale parameter $\theta$, then $Y=\log (T)$ has a Type I extreme-value distribution 
with location parameter $a=\log (\theta)$ and scale parameter $b=1 / \beta$. Consequently, if $\hat{a}$ and $\hat{b}$ are estimates of $a$ and $b$, then $\hat{\theta}=\exp (\hat{a})$ and $\hat{\beta}=1 / \hat{b}$ are estimates of $\theta$ and $\beta$, respectively.

These remarks also apply with the exponential distribution because it is a special case of the Weibull distribution with $\beta=1$. Thus, if $T$ is exponential with scale parameter $\theta=1 / \lambda$, then $Y=$ $\log (T)$ has a Type I extreme-value distribution with parameters $a=\log (\theta)$ and scale parameter $b=1$.

\section{Estimation Using SAS}

As noted previously, it is possible to use the SAS Procedure PROC LIFEREG to obtain estimates of the parameters of some of the models discussed here. However, it is necessary to know about how this procedure handles the data. It is required to specify a model. The models allowed include exponential, Weibull, normal and lognormal, along with some others which we will not discuss. The procedure automatically performs a logarithmic transformation on the data. For example, if Weibull is specified as the model, PROC LIFEREG converts the data to Type I extreme-value and estimates the parameters for that model. Similarly, lognormal data is converted to normal data when the lognormal model is specified.

In case the data has already had a logarithmic transformation, there is a NOLOG option which can be used in the model step prior to specifying the distribution. For example, if the data is normal data and it is desired to analyze it with PROC LIFEREG, one can invoke the NOLOG option and the lognormal model. The program will compute the mean and standard deviation of the data. When the NOLOG option is not used with exponential, Weibull or lognormal data, the resulting estimates are for the transformed model and must be converted back to the original model using the results discussed above.

For example, if the Weibull model is specified with the outage time data of Example 1, the program uses a log-transform and computes the location and scale estimates 3.8996 for $a$ and 0.2040 for $b$ which can be used to compute estimates of the Weibull parameters as $\hat{\beta}=1 / 0.2040=4.90$ and $\hat{\theta}=$ $\exp (3.8996)=49.4$. On the SAS printout the estimate of location, $\hat{a}$, is the variable called INTERCPT, and the estimate of scale, $\hat{b}$, is the variable called SCALE. These terms correspond to the graphical interpretation for probability plots described above. 


\section{Repairable Systems}

The preceding discussion assumes that a unit such as a system or component is observed only once or else that several independent copies of the unit are observed, resulting in a random sample of duration times. For instance, in Example 1 we modeled refueling outage times for a plant as if they were independent and identically distributed measurements. In reality, we were measuring the same plant repeatedly, but assuming that the conditions affecting outage times are the same each time, and that the distributions of later measurements are unaffected by what was observed earlier. For example, if an outage time is larger than average, this does not increase the likelihood that next one observed will behave this way. In this case, a trend in the outage times would not be expected. However, there could be circumstances under which repeated measurements would exhibit a trend in time.

\section{Analysis of Trends}

With events observed repeatedly over time, changes in the rate of occurrence can happen. Methods for analyzing trends over time are common in applications involving the reliability of repairable systems, and the methods will be discussed in this context. However, these methods can also be used in any application where the same type of event is observed repeatedly over time.

With some repairable systems, instead of replacing a failed unit, a less than perfect repair is made and the unit is placed back into service. In this case, one might expect a trend in which times between failures tend to be shorter with the passage of time, reflecting deterioration of the system with age. On the other hand, there are situations in which an extensive analysis is done to determine the failure mode, and if possible corrective action is taken to make this failure mode less likely to occur during subsequent operation of the unit. The corrective action might consist of replacement of a failed part with one of a better design, or a part made of more durable material. This situation is generally known as reliability growth.

A successful program of reliability growth will result in a tendency toward longer times between failures over time. Consider, for example, the successive times of critical compressor failures discussed in Example 2. As noted previously, the critical failures of the compressor are those which result in a shutdown of the compressor while repairs are made. If failure modes can be identified and corrective measures are implemented, reducing the likelihood of recurrence of such failure modes in the future, then one would expect an increasing trend in operating times between failures over time. One way to visualize such a trend is a plot of the number of failures $N(t)$ versus operating time $t$ of the compressor. Such a plot based on the data of Example 2 are shown in Figure 16. 


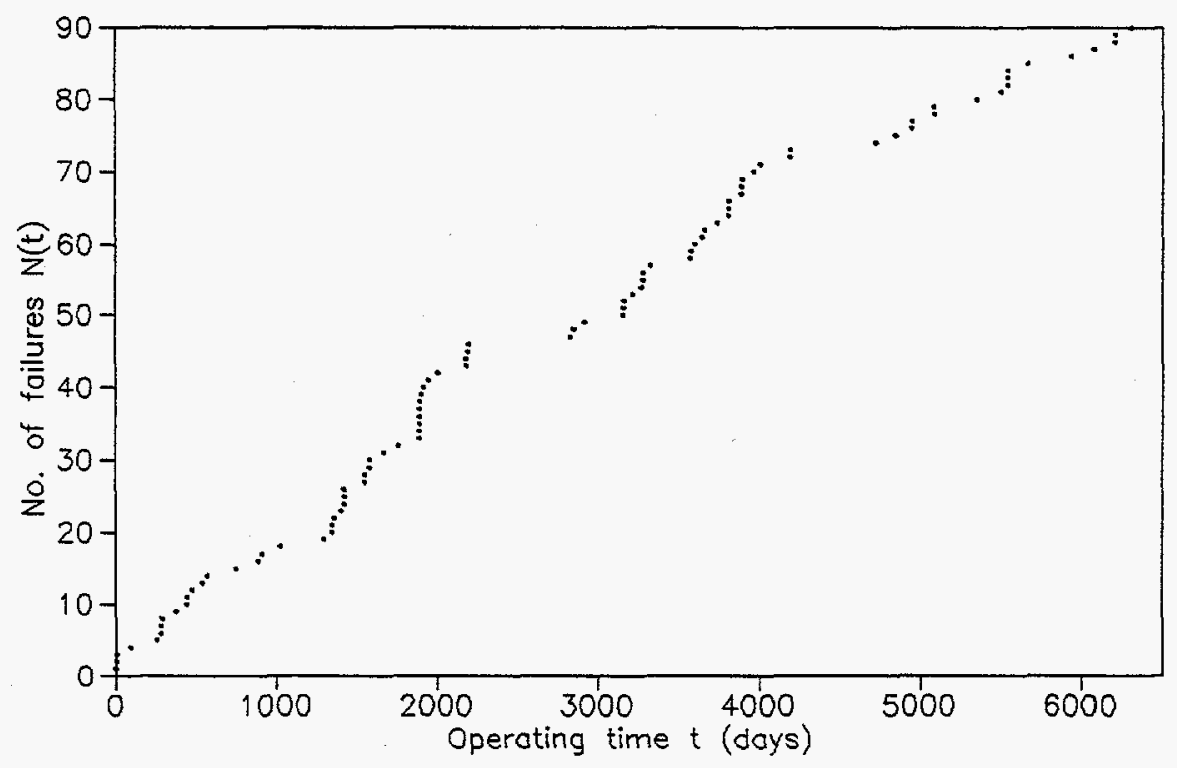

Figure 16. Number of critical compressor failures as a function of times (operating days).

An examination of Figure 16 reveals a slight concave downward pattern in the plotted points. Such a pattern would be expected in the case of reliability growth because the rate at which failures are occurring will tend to decrease over time. Although a plot of this sort is useful as a diagnostic tool, it is desirable to have a formal test which is capable of determining the whether an observed trend is statistically significant.

A popular method for testing reliability growth is based on the assumption that for each time $t$, the number of failures $N(t)$ has a mean value of the form

$\mathrm{E}[N(t)]=(t / \theta)^{\beta}$

where $\theta$ and $\beta$ are respectively scale and shape parameters. Under certain conditions, as discussed by Bain and Engelhardt (1992, page 415), $N(t)$ is Poisson distributed and is called the Power Law process.

If $\beta>1$, the mean value, $\mathrm{E}[N(t)]$, is a concave upward function of $t$; If $\beta=1$ the mean value is linear with slope $1 / \theta$ and intercept 0 ; When $\beta<1$ the mean value is a concave downward function 
of $t$. Thus, statistical evidence suggesting $\beta<1$ corresponds to reliability growth, and $\beta>1$ corresponds to deterioration of the system. We will denote the first $n$ cumulative operating times as $X_{1}$, $X_{2}, \ldots, X_{n}$, and assume that observation was stopped after a fixed number of failures $n$. Under this assumption the ML estimates of the parameters can be derived, and the resulting estimates are

$\hat{\beta}=n / \operatorname{In}\left(X_{n} / X_{i}\right)$

where the summation on $i$ ranges from 1 to $n$, and

$\hat{\theta}=X_{n} / n^{1 / \hat{\beta}}$

For example, based on the data from Example 2, we have that $\hat{\beta}=0.76$ and $\hat{\theta}=16.95$. The fact that $\hat{\beta}<1$ is consistent with the earlier observation that the plot in Figure 16 has a concave downward shape with a pattern which is characteristic of reliability growth.

Lower and upper limits for a $(1-2 \alpha) 100 \%$ confidence interval for $\beta$ are, respectively

$\beta_{\mathrm{L}}=\hat{\beta} \chi_{2(n-1), \alpha}^{2} /(2 n)$

and

$\beta_{U}=\hat{\beta} \chi_{2(n-1), 1-\alpha}^{2} /(2 n)$.

The upper confidence limit $\beta_{U}$, when considered individually, defines a $(1-\alpha) 100 \%$ upper onesided confidence interval for $\beta$. In other words, we are $(1-\alpha) 100 \%$ confident that $0<\beta \leq \beta_{\mathrm{U}}$. If $\beta_{\mathrm{U}}<1$, there is evidence at the $\alpha$ level of significance that $\beta<1$. For the data of Example 2, limits for a $90 \%$ confidence interval are $\beta_{\mathrm{L}}=0.63$ and $\beta_{\mathrm{U}}=0.89$. The upper confidence limit, $\beta_{\mathrm{U}}$, holds individually with $95 \%$ confidence, and the fact that it is less than 1 is evidence of a trend toward longer operating times with $\alpha=0.05$ level of significance.

As mentioned above, a trend toward shorter times between successive failures might be observed in a deteriorating system, and an approach similar to that used above can be used to test for this situation. An increasing rate of occurrence of failures would be expected in a deterioration system, corresponding to $\beta>1$ for the Power Law model. In this case, statistically significant results are obtained, at significance level $\alpha$, when $1<\beta_{\mathrm{L}}$, the limit for a lower one-sided $(1-\alpha) 100 \%$ confidence interval.

It is possible to use some of the results discussed earlier about exponential distributions in order 
to test the goodness-of-fit of the Power Law process. In particular, as noted by Bain and Engelhardt (1992, page 428), the $n-1$ transformed variables $Y_{1}, Y_{2}, \ldots, Y_{n-1}$ such that

$Y_{i}=\ln \left(X_{n} / X_{n-i}\right)$

are distributed the same as order statistics in a random sample of size $n-1$ from an exponential distribution with mean $1 / \beta$. Consequently, the CVM test for the exponential model, applied to the $Y_{i} s$ provides a test for the goodness-of-fit of the Power Law process to the original failure data. The CVM statistic for the transformed data of Example 2 is $C M=0.121$. From Table 10 of Bain and Engelhardt (1992), the 0.90th quantile is $C M_{0.90}=0.177$, indicating a reasonable fit for the model.

Other statistical methods for analyzing data from a Power Law model can be found in Bain and Engelhardt (1991, Ch. 9).

It should be noted that two other tests for trend are discussed by Engelhardt (1994). One of these tests, called the Laplace test, was derived under the assumption, that the mean value at time $t$ is of the form $\mathrm{E}[N(t)]=\exp (a+b t)$. However, for the data of Example 2, this model does not fit nearly as well as the Power Law process, and the above method is preferred. The other test discussed by Engelhardt (1994), called the Mann test, is based on the ranks of successive times between failures, and it requires weaker assumptions than the Laplace test or the test discussed above for the Power Law model. However, the test discussed above is recommended when use of the Power Law model is indicated.

\section{Availability and Maintainability}

In the discussion which follows, we will consider a system consisting of one or more components which, when the components fail, are either replaced with a brand new one or repaired to "like-new" condition. Thus, we have a sequence of uptimes $T_{1}, T_{2}, \ldots$ for the system. In other words, initially the system is operational or "up" until the first failure occurs a time denoted by $T_{1}$. Then, each time the system fails, it is repaired and restarted, and we denote by $T_{i}$ the time between the (i - 1)st restart and the $i$ th failure. These variables are modeled as random variables, and they represent absolute differences of between times when a system is restarted and the next failure.

We will assume that the uptimes $T_{1}, T_{2}, \ldots$ are independent and each has the same distribution, say $F_{T}(t)=P\left[T_{i} \leq t\right]$, and the same $\mathrm{E}(T)$. Similarly, when a system fails it will be "down" for a certain period of time, called the downtime or repair time. If the downtimes are negligible relative to the uptimes, then they are usually ignored. However, if the downtimes are not negligible, the analysis of the system should take them into account. 
As in the case of uptimes, we have a sequence of downtimes, denoted by $D_{1}, D_{2}, \ldots$, which are assumed to be independent random variables with the same distribution, $F_{D}(t)=P\left[D_{i} \leq t\right]$, and the same mean time to repair (MTTR). The CDF $F_{D}(t)$ is called the maintainability of the system (see e. g. Lewis, 1987, p. 266). The $D_{i}$ s represent absolute differences between times when a system fails and subsequent restarts. Downtimes are usually affected by a number of factors such as

- access time

- diagnosis time

- repair (or replacement) time

- checkout time

- time waiting for service or replacement parts

It is possible to define a function which is analogous to the hazard function, sometimes called the instantaneous repair rate,

$$
h_{D}(t)=\frac{f_{D}(t)}{1-F_{D}(t)}
$$

where $f_{D}(t)$ is the pdf of $D$. Analogous with the case of the hazard function, downtimes are exponentially distributed if and only if the corresponding repair rate is constant, say $h_{D}(t)=\nu$.

A convenient way to model the status of a repairable system is in terms of a state variable, defined as $S(t)=1$ if the system is up at time $t$, and $S(t)=0$ if it is down at time $t$. The availability of a system at time $t$ is the probability that the system is up at time $t$,

$A(t)=P[S(t)=1]$

and the unavailability is $U(t)=P[S(t)=0]$, or

$U(t)=1-A(t)$.

Actually, this same idea can be used to unify applications involving both repairable and nonrepairable units. In the case of units which are nonrepairable and discarded when they fail, $S(t)=$ 
1 if the unit is still operating at time $t$, and $S(t)=0$ if it has failed by time $t$. If $T$ is the time of failure, then $S(t)=1$ if and only if $T>t$, which means that for nonrepairable units availability and reliability are the same thing, $A(t)=R(t)$. However, with repairable systems the situation is somewhat more complicated. We will consider several different cases. In each of the following cases, we assume independence not only within sequences of uptimes $\left\{T_{\mathrm{i}}\right\}$ and downtimes $\left\{D_{\mathrm{i}}\right\}$, but also independence between uptimes and downtimes. That is, all random variables in the sequence $T_{1}, D_{1}, T_{2}, D_{2}, \ldots$ of uptimes and downtimes are independent.

Consider a repairable system where the uptimes are exponential with failure rate $\lambda$ and the downtimes are exponential with repair rate $\nu$. The MTTF and MTTR are $1 / \lambda$ and $1 / \nu$, respectively. It can be shown (see e. g. Lewis, 1987, p. 270), with these assumptions, that

$$
A(t)=\frac{v}{\lambda+v}+\frac{\lambda}{\lambda+v} \mathrm{e}^{-(\lambda+v) t}
$$

for all $t \geq 0$. Thus, $A(0)=1$ and

$$
\lim _{t \rightarrow \infty} A(t)=\frac{v}{\lambda+v}=\frac{1 / \lambda}{1 / \lambda+1 / v}=\frac{\text { MTTP }}{\text { MTTF }+ \text { MTTR }}
$$

In areas such as Probabilistic Risk Assessment (PRA) it is common practice to think of availability and unavailability as constant quantities, free of time. For a new system these quantities with depend on the operating time, but with increasing time the dependence is slight, and the limiting value given in Equation (5) can be used. Also, if MTTR $=\infty$ (or $\nu=0$ ) is interpreted as not repairing the system when it fails, then the availability is the same as the reliability,

$$
A(t)=R(t)=\exp (-\lambda t)
$$

The availability function and limiting availability for a repaired system and the reliability function for a nonrepaired system are shown in Figure 17. As noted above, the reliability can be viewed as the availability in the situation where the system fails once and is not subsequently repaired. With this interpretation, it is not surprising that the availability would drop to zero as $t$ approaches $\infty$. On the other hand, if the system is repaired when it fails, the availability approaches the positive limit on the right side of (5).

The results on availability discussed above are based on a rather strong assumption about the distributions of the uptimes and downtimes, namely exponentiality. It can be shown, using a branch of probability theory known as renewal theory that the limit given by (5) is valid under very general 


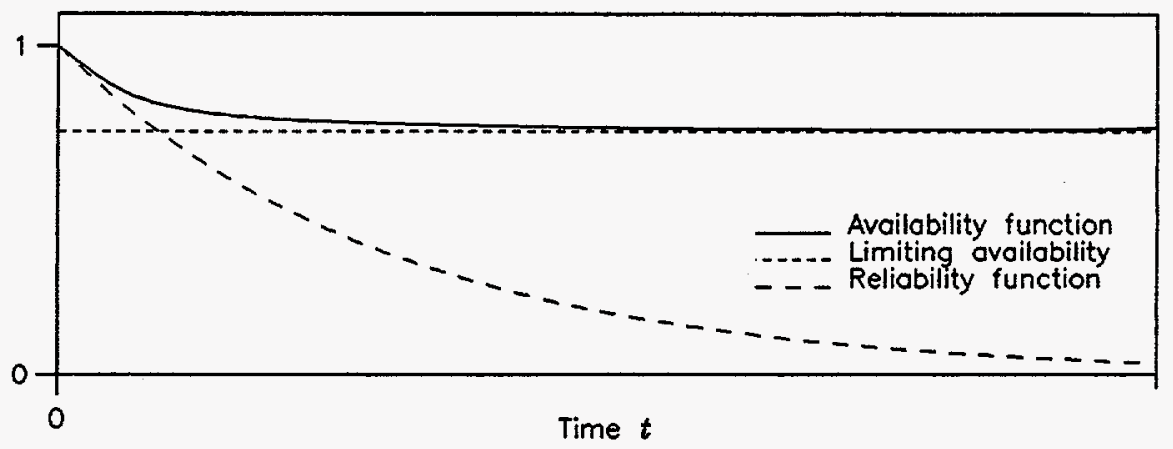

Figure 17. Comparison of availability for repaired system and nonrepaired system.

conditions. For a complete discussion of these results see Ross (1983, pages 66-67).

As an example, we consider the uptimes and downtimes of Example 3. The status variable $S(t)$ is plotted in Figure 18, where time is measured in days.

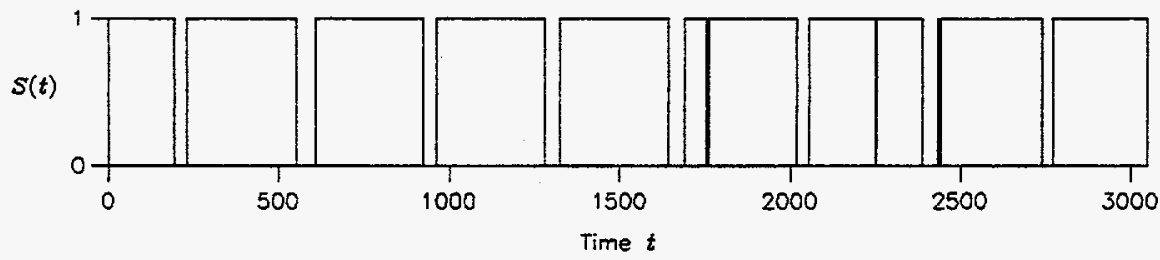

Figure 18. Uptime and downtime status for Plant A during the period 1985-94.

The limiting availability can be estimated by first estimating MTTF by $\bar{t}=224.3$ days and MTTR by $\bar{d}=32.2$ days. Thus, an estimate of the limiting availability is $\hat{A}(\infty)=224.3 /(224.3+$ 32.2) $=0.87$. 


\section{REVIEW}

This section summarizes the tools that have been presented in this report.

Models and methods for analyzing time data were considered in this report. Many key results involving random times are motivated by the study of lifetimes, and therefore much of the report focuses on such results. For lifetimes of people or organisms, such analysis is usually called survival analysis, and for failure times of hardware, software or systems, it is called reliability. Although the report tends to emphasize results in reliability, the analysis tools discussed here also apply to more general studies of duration times.

Observed times, obtained through experiments, studies or reviews of operational data, were modeled as random quantities. An example of this is the time to failure or lifetime of a unit such as a component or system. Typically, the time to failure is subject to chance variations such as design flaws or manufacturing defects in the hardware, human errors from installation or operation of equipment, or possibly environmental reasons. It is therefore natural to interpret the observed time as a positive-valued random variable $T$. Similarly, other duration times such as repair times are subject to chance variations and, thus, modeled as random variables.

As noted previously, there are different ways to specify a probability distribution, and the most common way is to specify the probability density function (pdf). Other characteristics of interest are descriptive quantities such as the distribution mean, variance or quantiles. Although nonparametric estimates are easily computed using data from a random sample, more efficient estimates can be obtained by fitting a parametric model by the method of maximum likelihood.

Several models are commonly considered in a study of time data. The models considered in this report were

\section{- Exponential}

- Weibull

- $\quad$ Gamma

- $\quad$ Normal

- Lognormal 
The exponential, Weibull, normal and lognormal models have the advantage that they either they involve only location and scale parameters, or models obtained using logarithm transformations involve only location and scale parameters. Consequently, standard fitting procedures such as probability plots are fairly straightforward, and the intercepts and slopes of linear fits to these plots can be used to obtain rough estimates of the location and scale parameters. The gamma model doesn't have this advantage. The normal model has the disadvantage that there is a nonzero probability of a negative value of time. However, if the ratio of the mean to the standard deviation is sufficiently large, this probability is negligible. On the other hand, the other models assign zero probability to the negative portion of the horizontal axis.

The process of fitting a model typically involves exploratory graphical methods followed by formal confirmation by means of a goodness-of-fit test. Two graphical methods discussed in this report are hazard plotting and probability plotting. Two goodness-of-fit tests considered are the ordinary chisquare test and the Cramér-Von Mises (CVM) test. Either test can be used to test whether a hypothesized model fits, but the CVM test is more convenient to compute, provided that the appropriate tables of quantiles are readily available.

In the preceding discussion it was assumed that when repeated observations are made on units of the same type, the values obtained can be modeled as data from a random sample. For example, in Example 1 we modeled refueling outage times for either Plant A or Plant B as if they were independent and identically distributed. In reality, we were measuring the same unit repeatedly over time, but assuming that the conditions affecting outage times are the same each time, and that the distributions of later measurements are unaffected by what was observed earlier. For example, if an outage time is larger than average, the next one observed is not affected in a similar way.

There are circumstances where repeated measurements will be dependent and possibly exhibit some sort of trend in time. For example, it is often the case that instead of replacing a failed unit, a minimal repair is made, and when it is placed back into service it is no longer as reliable as a new unit. In this case, we might expect a trend in which times between failures tend to be shorter with the passage of time. The analysis of situations such as this make use of tests based on special models such as the Power Law model to determine the presence of a significant trend.

In some situations it is necessary to consider two types of durations which alternate back and forth in time, such as uptimes and downtimes of a system such as a nuclear power plant. The model for uptimes is the exponential distribution discussed earlier. For the downtimes, a function is defined which is analogous to the hazard function, and is called the instantaneous repair rate. Analogous to the assumption of constant hazard function for uptimes, a constant repair rate implies that downtimes are 
exponentially distributed.

For any point in time $t$, such a system is either up or down. The availability $A(t)$ of a system at time $t$ is the probability that the system is up at time $t$, and the unavailability, $U(t)=1-A(t)$ is the probability that the system is down at time $t$. If the uptimes and downtimes are exponentially distributed, an exact mathematical expression can be derived for availability of a repairable system. Otherwise, it is possible under fairly general assumptions to approximate the availability for a system that has been operating for a sufficiently long period of time. In particular, if uptimes and downtimes are all independent, and the mean uptimes and mean downtimes are, respectively, MTTF and MTTR, then the approximate availability is MTTF/(MTTF + MTTR). Because MTTF and MTTR are means of the respective distributions of uptimes and downtimes, they are easily estimated from operational data. 


\section{REFERENCES}

Ascher, H., and Feingold, H., 1984, Repairable Systems Reliability, New York: Marcel Dekker.

Atwood, C. L., 1994, Hits per Trial: Basic Analysis of Binomial Data, EGG-RAAM-11041.

Atwood, C. L., 1995, Modeling Patterns in Count Data Using Loglinear and Related Models, INEL-950121.

D’Agostino, R. B., and Stephens, M. A., 1986, Goodness-of-Fit Techniques, New York: Marcel Dekker.

Engelhardt, M. E., 1994, Events in Time: Basic Analysis of Poisson Data, EGG-RAAM-11088.

Bain, L. J., and Engelhardt, M., 1991, Statistical Analysis of Reliability and Life-Testing Models: Theory and Methods, Second Edition, New York: Marcel Dekker.

Bain, L. J., and Engelhardt, M., 1992, Introduction to Probability and Mathematical Statistics, Second Edition, Boston: PWS-Kent.

Høyland, A., and Rausand, M., 1994, System Reliability Theory, New York: John Wiley and Sons.

Kaplan, E. L. and Meier, P., 1958, Nonparametric estimation from incomplete observations, Journal of the American Statistical Association, 53, p. 457-481.

Lewis, E. E., 1987, Introduction to Reliability Engineering, New York: John Wiley and Sons.

Nelson, W., 1982, Applied Life Data Analysis, New York: John Wiley and Sons.

Ross, S., 1983, Stochastic Processes, New York: John Wiley and Sons.

Sweet, A. L. 1990, On the Hazard Rate of the Lognormal Distribution, IEEE Transactions on Reliability, 39, pp. 325-328.

WASH-1400. 1975, Reactor Safety Study, U.S. Nuclear Regulatory Commission, NUREG-75/014. 
Appendix A: SAS Programs

A-1 
A-2 


\section{Appendix A}

\section{SAS Programs}

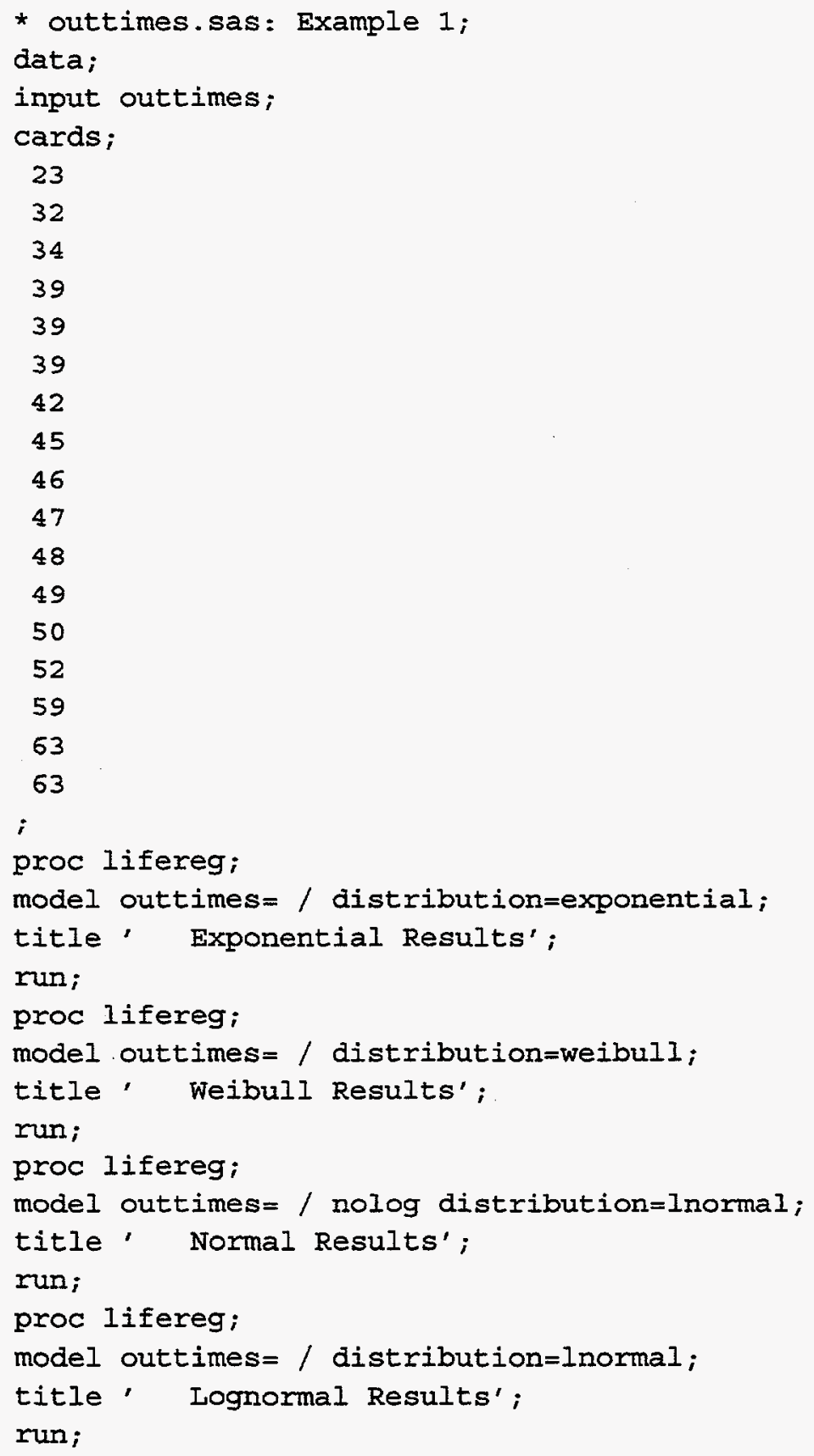




\section{Output}

Exponential Results

$10: 13$

Tuesday, August 1, 19951

L I F E R E G P R O C E D U R E

Data set $\quad=$ WORK. DATA1

Dependent Variable=Log (OUTTIMES)

Noncensored Values $=17$ Right Censored Values $=\quad 0$

Left Censored Values $=0$ Interval Censored Values $=0$

Log Likelihood for EXPONENT -17.49252029

Exponential Results

$10: 13$

Tuesday, August 1, 19952

L I F E R E G P R O C E D U R E

Variable DF Estimate std Err Chisquare Pr>Chi Label/Value

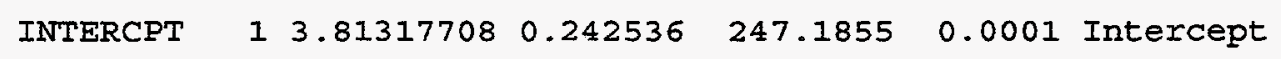

SCALE $\quad 0 \quad 1 \quad 0 \quad$ Extreme vaIue scale parameter

Iagrange Multiplier Chisquare for scale

- Pr>Chi is : .

Weibull Results

$10: 13$

Tuesday, August 1, 19953

L I F E R E $G R O C E D U R E$

Data set =WORK.DATA1

Dependent Variable=Log (OUTTIMES)

Noncensored Values $=17$ Right Censored Values $=\quad 0$

Left censored Values $=0$ Interval Censored Values $=0$

Log Likelihood for WEIBULI 0.4089200922

Weibull Results

$10: 13$

Tuesday, August 1, 19954

L I FERE G P ROCEDUR E

Variable DF Estimate std Err Chisquare Pr>Chi Label/Value

$\begin{array}{lllllll}\text { INTERCPT } & 1 & 3.89957188 & 0.052212 & 5578.25 & 0.0 & \text { Intercept }\end{array}$

SCALE $\quad 10.204027520 .038328 \quad$ Extreme value scale parameter 
Normal Results

$10: 13$

Tuesday, August 1, 19955

I I F E R E G P R O C E D U R E

Data set $\quad$ =WORK. DATAI

Dependent Variable=OUTTIMES

Noncensored Values $=17$ Right Censored Values $=\quad 0$

Ieft Censored Values $=0$ Interval Censored Values $=0$

Log Likelihood for NORMAL -63.92714816

Normal Results

$10: 13$

Tuesday, August 1, 19956

I I F E R E G P R O C E D U R E

Variable DF Estimate std Err Chisquare Pr>Chi Label/Value

INTERCPT $\quad 145.2941176 \quad 2.521554 \quad 322.6615 \quad 0.0001$ Intercept

SCALE $\quad 110.39663241 .783008 \quad$ Normal scale parameter

Lognormal Results

$10: 13$

Tuesday, August 1, 19957

I I F E R E G P R O C E D U R E

Data set

$=$ WORK.DATAI

Dependent Variable=Log (OUTTIMES)

Noncensored Values = 17 Right Censored Values $=\quad 0$

Left Censored Values $=0$ Interval Censored Values $=0$

Log Likelihood for LNORMAI -0.442069748

Lognormal Results

$10: 13$

Tuesday, August 1, 19958

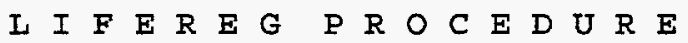

Variable DF Estimate std Err Chisquare Pr>Chi Label/Value

$\begin{array}{lllllll}\text { INTERCPT } & 1 & 3.78420539 & 0.060233 & 3947.17 & 0.0 & \text { Intercept }\end{array}$

SCALE $\quad 10.248345480 .042591 \quad$ Normal scale parameter 
* emp_surv.sas: Example 1;

title 'Empirical Survival Function';

data;

input outtimes;

cards ;

23

32

34

39

39

39

42

45

46

47

48

49

50

52

59

63

63

i

proc lifetest plots $=(s, 1 s, 11 s)$;

time outtimes;

run; 


\section{Output}

Empirical Survival Function

11:13 Tuesday, August 1, 19951

Product-Iimit Survival Estimates

\begin{tabular}{|c|c|c|c|c|c|}
\hline OUTTIMES & Survival & Failure & $\begin{array}{c}\text { Survival } \\
\text { Standard } \\
\text { Error }\end{array}$ & $\begin{array}{l}\text { Number } \\
\text { Failed }\end{array}$ & $\begin{array}{c}\text { Number } \\
\text { Left }\end{array}$ \\
\hline 0.0000 & 1.0000 & 0 & 0 & 0 & 17 \\
\hline 23.0000 & 0.9412 & 0.0588 & 0.0571 & 1 & 16 \\
\hline 32.0000 & 0.8824 & 0.1176 & 0.0781 & 2 & 15 \\
\hline 34.0000 & 0.8235 & 0.1765 & 0.0925 & 3 & 14 \\
\hline 39.0000 & . & . & - & 4 & 13 \\
\hline 39.0000 & . & . & . & 5 & 12 \\
\hline 39.0000 & 0.6471 & 0.3529 & 0.1159 & 6 & 11 \\
\hline 42.0000 & 0.5882 & 0.4118 & 0.1194 & 7 & 10 \\
\hline 45.0000 & 0.5294 & 0.4706 & 0.1211 & 8 & 9 \\
\hline 46.0000 & 0.4706 & 0.5294 & 0.1211 & 9 & 8 \\
\hline 47.0000 & 0.4118 & 0.5882 & 0.1194 & 10 & 7 \\
\hline 48.0000 & 0.3529 & 0.6471 & 0.1159 & 11 & 6 \\
\hline 49.0000 & 0.2941 & 0.7059 & 0.1105 & 12 & 5 \\
\hline 50.0000 & 0.2353 & 0.7647 & 0.1029 & 13 & 4 \\
\hline 52.0000 & 0.1765 & 0.8235 & 0.0925 & 14 & 3 \\
\hline 59.0000 & 0.1176 & 0.8824 & 0.0781 & 15 & 2 \\
\hline 63.0000 & . & . & - & 16 & 1 \\
\hline 63.0000 & 0 & 1.0000 & 0 & 17 & 0 \\
\hline
\end{tabular}

$\begin{array}{llllr}\text { Quantiles } & 75 \% & 50.0000 & \text { Mean } & 45.2941 \\ & 50 \% & 46.0000 & \text { Standard Error } & 2.5992 \\ & 25 \% & 39.0000 & & \end{array}$

Summary of the Number of Censored and Uncensored Values

Total Failed Censored scensored

$\begin{array}{llll}17 & 17 & 0 & 0.0000\end{array}$ 
Appendix B: Mathematical Details 
B-2 


\section{Appendix B}

\section{Mathematical Details}

\section{Estimation of Reliability from Censored Data}

If all of the data from a given study are available, then we say that it is a complete set of data. For example, if the data are obtained from a random sample of size $n$ and if all values $t_{1}, t_{2}, \ldots, t_{\mathrm{n}}$ are observed then the data set is called a complete sample. Another common situation is when some of the data are not observed. If the number of unobserved values is known, then we call the data censored. If the number unobserved is not known, then we call the data truncated. We will consider the case of censored data from a random sample.

There are many types of censoring possible, but we will discuss only cases in which the larger observations are missing. This is the most important case with lifetime data, because it is often necessary to begin an analysis before all data can be observed. For example, if a random sample of lifetimes are to be obtained from a test, it might be the case that most bulbs will have burned out within the first six months of the test, but the last few might continue to burn for another year or longer. It would be desirable to do an analysis based on those data which have been observed in the first six months, but it would be inappropriate to treat such censored data as if it were a complete sample because they only reflect behavior of "weaker" units in the population which tend to fail early.

If lifetimes are only observed prior to some fixed time $s$, then we have type I censoring on the right. The number of lifetimes observed is a random integer, say $r$. The other common type of censoring with life data is when a fixed number $r$ of lifetimes are observed. It is possible to express this in terms of the first $r$ observed order statistics, $t_{(1)}<t_{(2)}<\cdots<t_{(\mathrm{r})}$. These $r$ observations are said to have type $I I$ censoring from the right. This differs from type I censoring primarily in the fact that $r$ is fixed and the upper value $t_{(\mathrm{r})}$ is random, whereas with type I censoring the upper value $s$ is fixed and the number of lifetimes $r$ is part of the data. Thus, in the case of type I censoring $r$ is part of the data set. One of two things can occur with type I censoring, either no failures are observed before the censoring time $s$, or a nonzero number $r$ failure times are observed. When this second situation happens, it is convenient to use the same notation for the failure times as that used with type II censoring, namely $t_{(1)}$ $<t_{(2)}<\cdots<t_{(\mathrm{r})}$, although mathematically they must be handled differently.

There are also situations in which the $i$ th unit in the random sample might have a different fixed censoring time $s_{\mathrm{i}}$ for each $i$. That is, the $i$ th unit has associated with it a pair of values $\left(t_{\mathrm{i}}, s_{\mathrm{i}}\right)$, and what 
is observed is the smaller of the two, say $y_{\mathrm{i}}=\min \left(t_{\mathrm{i}}, s_{\mathrm{i}}\right)$. This is called a multiply censored sample, which agrees with the type I censored sample if $s_{\mathrm{i}}=s$ for all $i$. We can also define indicator variables of the form $\delta_{\mathrm{i}}=1$ if $t_{\mathrm{i}} \leq s_{\mathrm{i}}$, and 0 if $t_{\mathrm{i}}>s_{\mathrm{i}}$, and the resulting data set would consist of pairs $\left(y_{1}, \delta_{1}\right)$, $\left(y_{2}, \delta_{2}\right), \ldots,\left(y_{n}, \delta_{n}\right)$. Some common terminology which is helpful in understanding this situation is to call $t_{\mathrm{i}}$ the lifetime and $s_{\mathrm{i}}$ the "running time" of the $i$ th unit. In other words, we know that the $i$ th unit in the sample either failed at a known time $t_{\mathrm{i}}<s_{\mathrm{i}}$ or it was still running at time $s_{\mathrm{i}}$ and all that is known about the lifetime is $t_{\mathrm{i}}>s_{\mathrm{i}}$. Thus, $y_{\mathrm{i}}$ is either a lifetime or a running time, and $\delta_{\mathrm{i}}=1$ if it is a lifetime and 0 if it is a running time.

Analysis with censored data requires some modification of the methods for complete samples, because the larger lifetimes will not be known. The modification discussed here for multiply censored data is based on first estimating $\hat{R}(t)$ using an estimate known as the product-limit estimate. This estimate is also known as the Kaplan-Meier estimate because it was proposed by Kaplan and Meier (1958). The estimate is defined as follows: Consider the values $y_{1}, y_{2}, \ldots, y_{\mathfrak{n}}$ defined above for multiply censored data, and assume that they have been put in order, $y_{1}<y_{2}<\cdots<y_{\mathrm{n}}$. Let $J_{\mathrm{t}}$ represent the set of indices $j$ such that $y_{\mathrm{j}} \leq t$ and $y_{\mathrm{j}}$ is a lifetime (i.e. $\delta_{\mathrm{j}}=1$ ). Also denote by $n_{\mathrm{j}}$ the number of units still functioning at time $y_{\mathrm{j}}$ and $a_{\mathrm{j}}$ the number of units which fail at time $y_{\mathrm{j}}$. The product-limit estimate of $R(t)$ is

$$
\hat{R}(t)=\prod_{j \in J_{t}}\left(\frac{n_{j}-a_{j}}{n_{j}}\right) .
$$

When there is no censoring, the product-limit estimate of $R(t)$ turns out to be the same as $\hat{R}(t)=1$ $\hat{F}(t)$ where $\hat{F}(t)$ is the EDF.

The following estimation process, discussed in the framework of failure data, is due to Nelson (1972): As before, there are $n$ units which are observable, except when an associated censoring time occurs first. It is possible to compute an "empirical" estimate of $H(t)$ based on the failure data and the running times as follows: Suppose a failure is observed at time $t$. If $k$ units have survived until time $t$ (including the one which just failed), then $1 / k$ is the observed proportion failing at time $t$ among the $k$ units which have survived until time $t$. Consequently, $1 / k$ is an estimate of $h(t)$, and the sum of all such estimates for observed failure times $x \leq t$ is an estimate of $H(t)$.

With multiply censored data, the procedure is to rank failure times and running times together, but to include terms $1 / k$ in the estimate of $H(t)$ only for observed failure times, and not for running times. Neison's estimate of $H(t)$ is a first-order approximation to the estimate of $H(t)$ based on the product-limit estimate, but it is slightly easier to compute without special software. 


\section{REFERENCES}

Kaplan, E. L. and Meier, P., 1958, Nonparametric estimation from incomplete observations, Journal of the American Statistical Association, 53, p. 457-481.

Nelson, W., 1972, Theory and Application of Hazard Plotting for Censored Failure Data. Technometrics , 18, p. 105-114. 